\title{
Impact of generalized dissipative coefficient on warm inflationary dynamics in the light of latest Planck data
}

\author{
Abdul Jawad $^{1, \mathrm{a}}$, Shahzad Hussain ${ }^{2, \mathrm{~b}}$, Shamaila Rani ${ }^{1, \mathrm{c}}$, Nelson Videla ${ }^{3, \mathrm{~d}}$ \\ ${ }^{1}$ Department of Mathematics, COMSATS Institute of Information Technology, Lahore 54000, Pakistan \\ ${ }^{2}$ Department of Mathematics, Aspire College, Hafizabad, Pakistan \\ ${ }^{3}$ Instituto de Física, Pontificia Universidad Católica de Valparaíso, Avda. Universidad 330, Curauma, Valparaíso, Chile
}

Received: 26 April 2017 / Accepted: 24 September 2017 / Published online: 24 October 2017

(C) The Author(s) 2017. This article is an open access publication

\begin{abstract}
The warm inflation scenario in view of the modified Chaplygin gas is studied. We consider the inflationary expansion to be driven by a standard scalar field whose decay ratio $\Gamma$ has a generic power-law dependence with the scalar field $\phi$ and the temperature of the thermal bath $T$. By assuming an exponential power-law dependence in the cosmic time for the scale factor $a(t)$, corresponding to the intermediate inflation model, we solve the background and perturbative dynamics considering our model to evolve according to (1) weak dissipative regime and (2) strong dissipative regime. Specifically, we find explicit expressions for the dissipative coefficient, scalar potential, and the relevant inflationary observables like the scalar power spectrum, scalar spectral index, and tensor-to-scalar ratio. The free parameters characterizing our model are constrained by considering the essential condition for warm inflation, the conditions for the model evolves according to weak or strong dissipative regime, and the 2015 Planck results through the $n_{s}-r$ plane.
\end{abstract}

\section{Introduction}

Inflation is the most acceptable paradigm that describes the physics of the very early universe. Besides of solving most of the shortcomings of the hot big-bang scenario, like the horizon, the flatness, and the monopole problems [1-5], inflation also generates a mechanism to explain the largescale structure (LSS) of the universe [6-10] and the origin of the anisotropies observed in the cosmic microwave

\footnotetext{
a e-mails: abduljawad@ciitlahore.edu.pk; jawadab181@yahoo.com

b e-mail: shahzad_qau19@yahoo.com

c e-mails: shamailatoor.math@yahoo.com;

drshamailarani@ciitlahore.edu.pk

d e-mail: nelson.videla@pucv.cl
}

background $(\mathrm{CMB})$ radiation [11-18], since primordial density perturbations may be sourced from quantum fluctuations of the inlaton scalar field during the inflationary expansion.

The standard cold inflation scenario is divided into two regimes: the slow-roll and reheating phases. In the slowroll period the universe undergoes an accelerated expansion and all interactions between the inflaton scalar field and other field degrees of freedom are typically neglected. Subsequently, a reheating period $[19,20]$ is invoked to end the brief acceleration. After reheating, the universe is filled with relativistic particles and thus the universe enters in the radiation big-bang epoch. For a modern review of reheating, see [21]. On the other hand, warm inflation is an alternative mechanism for having successful inflation. The warm inflation scenario, as opposed to standard cold inflation, has the essential feature that a reheating phase is avoided at the end of the accelerated expansion due to the decay of the inflaton into radiation and particles during the slow-roll phase [2224]. During warm inflation, the temperature of the universe does not drop dramatically and the universe can smoothly enter into the decelerated, radiation-dominated period, which is essential for a successful big-bang nucleosynthesis. In the warm inflation scenario, dissipative effects are important during the accelerated expansion, so that radiation production occurs concurrently with the accelerated expansion. The dissipative effect arises from a friction term $\Gamma$ which describes the processes of the scalar field dissipating into a thermal bath via its interaction with other field degrees of freedom. The effectiveness of warm inflation may be parametrized by the ratio $R \equiv \Gamma / 3 H$. The weak dissipative regime for warm inflation is for $R \ll 1$, while for $R \gg 1$, it is the strong dissipative regime for warm inflation. Following Refs. [25,26], a general parametrization of the dissipative coefficient depending on both the temperature of the thermal bath $T$ and the inflaton scalar field $\phi$ can be written as 
$\Gamma(T, \phi)=C_{\phi} \frac{T^{m}}{\phi^{m-1}}$,

where the parameter $C_{\phi}$ is related with the dissipative microscopic dynamics and the exponent $m$ is an integer, where the value of the power $m$ dependent on the specifics of the model construction for warm inflation and on the temperature regime of the thermal bath. Typically, it is found that $m=3$ (low temperature), $m=1$ (high temperature) or $m=0$ (constant dissipation). Additionally, thermal fluctuations during the inflationary scenario may play a fundamental role in producing the primordial fluctuations $[27,28]$. During warm inflationary scenario the density perturbations arise from thermal fluctuations of the inflaton and dominate over the quantum ones. In this form, an essential condition for warm inflation to occur is the existence of a radiation component with temperature $T>H$, since the thermal and quantum fluctuations are proportional to $T$ and $H$, respectively [22,23,27-31]. When the universe heats up and becomes radiation dominated, inflation ends and the universe smoothly enters in the radiation Big-Bang phase [22,23]. For a comprehensive review of warm inflation, see Refs. [32,33].

The observational data from the luminosity-redshift of type Ia supernovae (SNIa), large-scale structure (LSS), and the cosmic microwave background (CMB) anisotropy spectrum, have supported evidence that our universe has started recently a phase of accelerated expansion [11-18,34-37]. The responsible for this acceleration of the late expansion is an exotic component having a negative pressure, usually known as dark energy (DE). Several models have been already proposed to be DE candidates, such as cosmological constant [38], quintessence [39-41], k-essence [4244], tachyon [45-47], phantom [48-50], Chaplygin gas [51], holographic dark energy [52], among others in order to modify the matter sector of the gravitational action. Despite the plenty of models, the nature of the dark sector of the universe, i.e. dark energy and dark matter, is still unknown. There exists another way of understanding the observed universe in which dark matter and dark energy are described by a single unified component. Particularly, the Chaplygin gas [51] achieves the unification of dark energy and dark matter. In this sense, the Chaplygin gas behaves as a pressureless matter at early times, and like a cosmological constant at late times. The original Chaplygin gas is characterized by an exotic equation of state with negative pressure,

$p_{c g}=-\frac{B}{\rho_{c g}}$,

whit $B$ being a constant parameter. The original Chaplygin gas has been extended to the so-called generalized Chaplygin (GCG) gas with the following equation of state [53]:

$p_{g c g}=-\frac{B}{\rho_{g c g}^{\lambda}}$, with $0 \leq \lambda \leq 1$. For the particular case $\lambda=1$, the original Chaplygin gas is recovered. The main motivation for studying this kind of model comes from string theory. The Chaplygin gas emerges as an effective fluid associated with D-branes which may be obtained from the Born-Infeld action [54]. At background level, the GCG is able to describe the cosmological dynamics [55], however, the model presents serious issues at the perturbative level [56]. Thus, a modification to the GCG, resulting in the modified Chaplygin gas (MCG) with a equation of state given by [57]

$p_{m c g}=A \rho_{m c g}-\frac{B}{\rho_{g c g}^{\lambda}}$,

where $A$ and $B$ are constant parameters, with $0 \leq \lambda \leq 1$, is suitable to describe the evolution of the universe [58,59] and it is also consistent with a perturbative study [60].

As we have seen, the original and generalized Chaplygin gas models are usually applied to explain the late-time acceleration of our universe as a possible candidate of dark energy. On the other hand, the modified Chaplygin gas (MCG) is also a model that mimics the behavior of matter at early times and that of a cosmological constant at late times. Given the attractiveness of the MCG as a dark energy candidate, a natural question to ask is: Can inflation be accommodated within the MCG scenario? This is the question we wish to address in the present work. However, we should emphasize that our inflationary model is not presented as a more desirable alternative to the conventional ones. Rather, we merely aim to establish the assumptions and extrapolations required to obtain successful inflation in a Chaplygin inspired model [61].

Various authors have examined warm inflation by considering the Chaplygin gas, standard and tachyon scalar field models in Einstein's General Relativity as well as in braneworld scenario with different expressions for the dissipative coefficient [62-77]. They found the consistency of their results with observational data, i.e., BICEP2, WMAP $(7+9)$ and Planck data. Moreover, many authors have investigated warm inflation in various alternative as well as modified theories of gravity [78-82]. Recently, Herrera et al. [83] studied warm intermediate inflation in the context of GCG in the weak and strong dissipative regimes by assuming a generalized form of the dissipative coefficient under slow-roll approximation. They found the constraints on the parameters by considering the Planck 2015 data, together with the essential condition for warm inflation $T>H$.

The main goal of the present work is to investigate the dynamics of warm inflation driven by a standard scalar field in the MCG scenario, with an inflaton decay rate $\Gamma$ given by the generalized expression of Eq. (1). By assuming an exponential power-law dependence in the cosmic time for the scale factor $a(t)$, we solve the background and pertur- 
bative dynamics considering our model to evolve according to $(1)$ weak dissipative regime $(R \ll 1)$ and $(2)$ according to strong dissipative regime $(R \gg 1)$. The free parameters characterizing our model are constrained by considering the essential condition for warm inflation, $T>H$, the condition for the model evolves according to weak or strong dissipative regime, and the 2015 Planck results through the $n_{s}-r$ plane.

This paper is organized as follows: in the next section, we present the basic setup of warm inflation in the MCG scenario. In Sects. 3 and 4, we solve the background and perturbative dynamics when the model evolves according to weak and strong regimes, respectively. Specifically, in each section, we find explicit expressions for the dissipative coefficient, scalar potential, and the relevant inflationary observables as the scalar power spectrum, scalar spectral index, and tensor-to-scalar ratio. Finally, Sect. 5 summarizes our finding and exhibits our conclusions. We have chosen units such that $c=\hbar=1$.

\section{Modified Chaplygin gas inspired inflation}

In this section, we introduce the basic setup of warm inflation in MCG scenario with a generalized expression for the inflaton decay rate $\Gamma$. As it was mentioned at the introduction, the exotic equation of state of MCG is given by

$p_{m c g}=A \rho_{m c g}-\frac{B}{\rho_{m c g}^{\lambda}}$,

where $A$ and $B$ are constant parameters with $0 \leq \lambda \leq 1$. $p_{m c g}$ and $\rho_{m c g}$ are the pressure and energy density of MCG, respectively. The energy density of MCG as a function of the scale factor $a$ can be obtained with the help of the stressenergy conservation law, yielding

$$
\begin{aligned}
\rho_{m c g} & =\left[\frac{B}{1+A}+\frac{C}{a^{3(1+\lambda)(1+A)}}\right]^{\frac{1}{1+\lambda}} \\
& =\rho_{m c g 0}\left[B_{S}+\frac{1-B_{S}}{a^{3(1+\lambda)(1+A)}}\right]^{\frac{1}{1+\lambda}},
\end{aligned}
$$

where $B_{s}=\frac{B}{1+A} \frac{1}{\rho_{m c g 0}^{1+\lambda}}, C$ is a positive integration constant. From the solution given by Eq. (6), the energy density of the MCG is characterized by three parameters, $B_{S}$ (or equivalently $B), A$, and $\lambda$. Particularly, in [84] by using a joint analysis of several tests at background as well as perturbative level, as the differential age of old galaxies, given by $H(z)$, baryonic acoustic oscillations (BAO) peak parameter, CMB shift parameter, SN Ia data, and growth index, the values for the best fit (with $\chi^{2} / d . o . f \sim 1.0296$ ) are given by $B_{s}=0.8252, A=0.0046$, and $\lambda=0.1905$.

As was mentioned in the introduction, in order to obtain successful inflation in a Chaplygin like inspired model, some assumptions and extrapolations are required. Following [61], we identify the energy density of matter $\rho_{m}$ with the contribution of the energy density associated to the standard scalar field $\rho_{\phi}$ through an extrapolation of Eq. (6), yielding

$$
\left[\frac{B}{1+A}+\rho_{m}^{(1+\lambda)(1+A)}\right]^{\frac{1}{1+\lambda}} \rightarrow\left[\frac{B}{1+A}+\rho_{\phi}^{(1+\lambda)(1+A)}\right]^{\frac{1}{1+\lambda}}
$$

In this sense, we will not consider Eq. (7) as a consequence of Eq. (6), but a non-covariant modification of gravity instead, resulting in a modifed Friedmann equation, as was pointed out in [85].

In this scenario, we consider a spatially flat universe which contains a self-interacting inflation field $\phi$ and a radiation field, then we write down a modified Friedmann equation of the form

$H^{2}=\frac{\kappa}{3}\left(\left[\frac{B}{1+A}+\rho_{\phi}^{(1+\lambda)(1+A)}\right]^{\frac{1}{1+\lambda}}+\rho_{\gamma}\right)$,

where $\kappa=8 \pi G$ and $H$ is the Hubble rate defined as $H=$ $\dot{a} / a$.

We recall that the Friedmann equation (8) constitutes a non-covariant modification of gravity. However, as was pointed out in Ref. [61], it may assumed that the effect giving rise to Eq. (8) preserves diffeomorphism invariance in $(3+1)$ dimensions, whence total stress-energy conservation follows. In this way, for our analysis, the second Friedmann equation is no longer required.

By coupling the inflaton field to a radiation fluid, the conservation equations for each individual component are given by [22-24]

$\dot{\rho}_{\phi}+3 H\left(\rho_{\phi}+P_{\phi}\right)=-\Gamma \dot{\phi}, \Longrightarrow \ddot{\phi}+3 H \dot{\phi}+V^{\prime}=-\Gamma \dot{\phi}$,

and

$\dot{\rho}_{\gamma}+4 H \rho_{\gamma}=\Gamma \dot{\phi}^{2}$

where $\rho_{\phi}=\frac{\dot{\phi}^{2}}{2}+V(\phi)$ and $P_{\phi}=\frac{\dot{\phi}^{2}}{2}-V(\phi)$ correspond to the energy density and pressure associated with the standard scalar field, respectively, and $V(\phi)$ is the inflaton's potential. On the other hand, $\Gamma$ represents the inflaton decay rate or dissipative coefficient, which is responsible for the process of decay of the scalar field into radiation during the inflationary expansion. This decay rate can be realized as a constant or can be a function of scalar field or temperature or both, i.e., $\Gamma(T, \phi)$. From first principles in quantum field theory this 
decay ratio $\Gamma$ has been already computed. A generalized form of $\Gamma$ is given by $[25,26]$

$\Gamma(T, \phi)=C_{\phi} \frac{T^{m}}{\phi^{m-1}}$.

In the literature, several cases have been studied for the different values of $m$, in special case $m=1$, i.e. $\Gamma \propto T$ represent high temperature SUSY case, for the value $m=0$ i.e. $\Gamma \propto \phi$ corresponds to an exponentially decaying propagator in the high temperature SUSY model, for $m=-1$ i.e. $\Gamma \propto \frac{\phi^{2}}{T}$, with non-SUSY case.

Considering that during warm inflation the energy density associated of radiation field $\rho_{\phi} \gg \rho_{\gamma}$ is subdominant with respect to energy density of the scalar field [22-24,27-31], Eq. (8) becomes

$$
\begin{aligned}
H^{2} & \approx \frac{\kappa}{3}\left(\left[\frac{B}{1+A}+\rho_{\phi}^{(1+\lambda)(1+A)}\right]^{\frac{1}{1+\lambda}}\right) \\
& =\frac{\kappa}{3}\left(\left[\frac{B}{1+A}+\left(\frac{\dot{\phi}^{2}}{2}+V(\phi)\right)^{(1+\lambda)(1+A)}\right]^{\frac{1}{1+\lambda}}\right) .
\end{aligned}
$$

By combining Eqs. (9) and (12), we obtain the square velocity of the inflaton field,

$$
\begin{aligned}
\dot{\phi}^{2}= & \frac{2(-\dot{H})}{\kappa(1+A)(1+R)}\left(\frac{3 H^{2}}{\kappa}\right)^{\frac{-A}{1+A}} \\
& \times\left[1-\frac{B}{1+A}\left(\frac{3 H^{2}}{\kappa}\right)^{-(1+\lambda)}\right]^{-\frac{A+\lambda(1+A)}{(1+A)(1+\lambda)}} .
\end{aligned}
$$

In this equation, we have introduced a new parameter $R$ defined by

$$
R \equiv \frac{\Gamma}{3 H} .
$$

This parameter measures the relative strength of thermal damping compared to the expansion damping. In warm inflation, two possible regimes can be described through $R$, i.e., weak dissipative regime in which $R \ll 1$ and Hubble damping is still the dominant term in this case. The second is strong dissipative regime which can be defined as $R \gg 1$ and $\Gamma$ controls the damped evolution of the inflation field.

By also assuming that $\dot{\rho}_{\gamma} \ll 4 H \rho_{\gamma}$, i.e., the radiation production is quasi-stable [22-24,27-31], Eqs. (10) and (13) lead to the relation for $\rho_{\gamma}$ as follows:

$$
\begin{aligned}
\rho_{\gamma}= & \frac{\Gamma \dot{\phi}^{2}}{4 H}=\frac{\Gamma(-\dot{H})}{2 \kappa H(1+A)(1+R)}\left(\frac{3 H^{2}}{\kappa}\right)^{\frac{-A}{1+A}} \\
& \times\left[1-\frac{B}{1+A}\left(\frac{3 H^{2}}{\kappa}\right)^{-(1+\lambda)}\right]^{-\frac{A+\lambda(1+A)}{(1+A)(1+\lambda)}} .
\end{aligned}
$$

In addition, the thermalized energy density of radiation field can be written as $\rho_{\gamma}=C_{\gamma} T^{4}$, where $C_{\gamma}=\pi^{2} g_{*} / 30$, and $g_{*}$ denotes the number of relativistic degrees of freedom. In particular, for the minimal supersymmetric standard model (MSSM), we have $g_{*}=228.75$ and $C_{\gamma} \simeq 70$ [26]. We can get the temperature of the thermal bath from Eq. (15) as follows:

$$
\begin{aligned}
T= & {\left[\frac{\Gamma(-\dot{H})}{2 \kappa C_{\gamma}(1+A)(1+R)}\right]^{1 / 4}\left(\frac{3 H^{2}}{\kappa}\right)^{\frac{-A}{4(1+A)}} } \\
& \times\left[1-\frac{B}{1+A}\left(\frac{3 H^{2}}{\kappa}\right)^{-(1+\lambda)}\right]^{-\frac{A+\lambda(1+A)}{4(1+A)(1 \lambda)}} .
\end{aligned}
$$

By considering Eqs. (12), (13) and (16), the inflaton's potential may be expressed as follows:

$$
\begin{aligned}
V= & {\left[\left(\frac{3 H^{2}}{\kappa}\right)-\frac{B}{1+A}\right]^{\frac{1}{(1+A)(1+\lambda)}} } \\
& +\frac{\dot{H}}{\kappa(1+A)(1+R)}\left(\frac{3 H^{2}}{k}\right)^{\frac{-A}{1+A}} \\
& \times\left[1-\frac{B}{1+A}\left(\frac{3 H^{2}}{\kappa}\right)^{-(1+\lambda)}\right]^{-\frac{A+\lambda(1+A)}{(1+A)(1+\lambda)}} .
\end{aligned}
$$

Similarly, by using Eqs. (11) and (16), the dissipative coefficient may be written as

$$
\begin{aligned}
\Gamma^{\frac{4-m}{4}}= & C_{\phi} \phi^{1-m}\left[\frac{(-\dot{H})}{2 \kappa C_{\gamma} H(1+A)(1+R)}\right]^{m / 4}\left(\frac{3 H^{2}}{\kappa}\right)^{\frac{-m A}{4(1+A)}} \\
& \times\left[1-\frac{B}{1+A} \times\left(\frac{3 H^{2}}{\kappa}\right)^{-(1+\lambda)}\right]^{-\frac{m(A+\lambda(1+A))}{4(1+A)(1+\lambda)}} \cdot(18)
\end{aligned}
$$

In the next two sections, we will explore the inflationary dynamics at background as well as perturbative level when our model evolves according to (1) weak dissipative regime and (2) strong dissipative regime, respectively. As an extra input, we assume an exponential power-law dependence in cosmic time for the scale factor $a(t)$, given by the intermediate inflation model. Exact solutions in the context of inflation can be found from an exponential potential, obtaining a solution for the scale factor give by $a(t) \sim t^{p}, p>1$, termed power-law inflation [86]. On the other hand, by considering a constant scalar potential [1], we obtain an exponential solution for the scale factor $a(t) \sim \exp H_{0} t$, known as de Sitter expansion. For an inverse power-law potential, the intermediate inflation model is found as an exact solution, for which the scale factor expands faster than power-law expansion but slower than de Sitter inflation. The scale factor $a(t)$ for intermediate inflationary model is given by [87]

$a(t)=\exp \left[\alpha t^{f}\right]$, 
where $\alpha>0$ and $0<f<1$. This model was in the beginning formulated as an exact solution to the background equations, nevertheless this model may be studied under the slow-roll approximation together with the cosmological perturbations [88-91].

\section{The weak dissipative regime}

Assuming that our model evolves according to the weak dissipative regime, i.e., $R \ll 1$ (or $\Gamma \ll 3 H$ ), the scalar field $\phi$ as a function of cosmic time may be found by using Eqs. (13) and (19), yielding

$\phi(t)-\phi_{0}=\frac{M[t]}{S}$,

where $\phi_{0}$ is a constant of integration, and the constant $S$ is given by

$S=\frac{A(2-f)+f}{\sqrt{2(1-f)(1+A)}}\left(\frac{1}{\alpha f}\right)^{\frac{1-A}{2(1+A)}}\left(\frac{\kappa}{3}\right)^{\frac{1-A}{2(1+A)}}$,

while $M[t]$ is a function of cosmic time taking the following form:

$$
\begin{aligned}
& M[t]=t^{\frac{f+2 A-A f}{2(1+A)}}{ }_{2} F_{1}\left[\frac{A(2-f)+f}{4(1+\lambda)(1+A)(1-f)},\right. \\
& \frac{A+\lambda(1+A)}{2(1+A)(1+\lambda)}, 1+\frac{A(2-f)+f}{4(1+\lambda)(1+A)(1-f)}, \\
&\left.\frac{B}{1+A} 3^{-(1+\lambda)} t^{2(1-f)(1+\lambda)}\left(\frac{\kappa}{f^{2} \alpha^{2}}\right)^{1+\lambda}\right],
\end{aligned}
$$

here ${ }_{2} F_{1}$ denotes the hypergeometric function [92] Under the slow-roll approximation, in which $\dot{\phi}^{2} / 2<V(\phi)$, from Eq. (17), the scalar potential as a function of scalar field can be written as

$$
V(\phi) \approx\left[\left(\frac{3 \alpha^{2} f^{2}}{\kappa\left(M^{-1}[S \phi]\right)^{2(1-f)}}\right)^{(1+\lambda)}-\frac{B}{1+A}\right]^{\frac{1}{(1+A)(1+\lambda)}} .
$$

In a similar way, we can obtain the dissipative coefficient in terms of the scalar field as

$$
\begin{aligned}
& \Gamma(\phi)=\left[\frac{1-f}{2 \kappa C_{\gamma}(1+A)\left(M^{-1}[S \phi]\right)}\right]^{\frac{m}{4-m}} \\
& \times\left(\frac{3 \alpha^{2} f^{2}}{\kappa\left(M^{-1}[S \phi]\right)^{2(1-f)}}\right)^{\frac{-m A}{(1+A)(4-m)}} \times C_{\phi}^{\frac{4}{4-m}} \phi^{\frac{4(1-m)}{4-m)}} \\
& \times\left[1-\frac{B}{1+A}\left(\frac{\kappa\left(M^{-1}[S \phi]\right)^{2(1-f)}}{3 \alpha^{2} f^{2}}\right)^{(1+\lambda)}\right]^{-\frac{m(A+\lambda(1+A))}{(1+A)(1+\lambda)(4-m)}}
\end{aligned}
$$

The number of $e$-folds, $N$, between two different values of cosmic time, $t_{1}$ and $t_{2}$, or equivalently, between two values of the scalar field, $\phi_{1}$ and $\phi_{2}$ is defined as follows:

$$
\begin{aligned}
N & =\int_{t_{1}}^{t_{2}} H d t=\alpha\left(t_{2}^{f}-t_{1}^{f}\right) \\
& =\alpha\left(\left(M^{-1}\left[S \phi_{2}\right]\right)^{f}-\left(M^{-1}\left[S \phi_{1}\right]\right)^{f}\right) .
\end{aligned}
$$

Since we are dealing with the scale factor $a(t)$, it is straightforward to use the slow-parameters

$\epsilon=-\frac{\dot{H}}{H^{2}}$,

and

$\eta=-\frac{\ddot{H}}{H \dot{H}}$.

In the intermediate inflation model, the slow-roll parameters $\epsilon$ and $\eta$ decrease as the field rolls down the potential, then there is no natural exit from the model [90]. However, from the definition of the parameter $\epsilon$, we may obtain the value of the scalar field for inflationary scenario at early stage $(\epsilon=1)$ [90], giving

$\phi_{1}=\frac{1}{S} M\left[\left(\frac{1-f}{\alpha f}\right)^{1 / f}\right]$.

In this way, we may evaluate the inflationary observables at $N e$-folds which have passed since the beginning of the inflationary period.

In the following, we will study the scalar and tensor perturbations for our warm inflation model in the MCG scenario, considering it to evolve according to the weak regime. For the case of the scalar perturbations, the amplitude could be stated as $\mathcal{P}_{\mathcal{R}}^{1 / 2}=\frac{H}{\dot{\phi}} \delta \phi$ [93]. Additionally, in the warm inflation scenario, a thermalized radiation component is present with $T>H$, then the inflaton fluctuations $\delta \phi$ are predominantly thermal instead quantum. Particularly, for the weak dissipation regime, the amplitude of the scalar field fluctuation was found to be $\delta \phi^{2} \simeq H T$ [27]. Then the power spectrum of the scalar perturbations can be obtained by utilizing Eqs. (13), (16), and (18):

$$
\begin{aligned}
\mathcal{P}_{\mathcal{R}}= & \frac{\sqrt{3}}{4} \kappa(1+A)\left(\frac{3}{\kappa}\right)^{\frac{(3-m) A}{(1+A)(4-m)}} \\
& \times\left[\frac{C_{\phi}}{2 \kappa C_{\gamma}(1+A)}\right]^{\frac{1}{4-m}} \phi^{\frac{1-m}{4-m}}(-\dot{H})^{\frac{m-3}{4-m}} \\
& \times(H)^{\frac{(11-3 m)(1+A)+2 A(3-m)}{(1+A)(4-m)}} \\
& \times\left[1-\frac{B}{1+A}\left(\frac{3 H^{2}}{\kappa}\right)^{-(1+\lambda)}\right]^{\frac{(3-m)[A+\lambda(1+A)]}{(4-m)(1+A)(1+\lambda)}} .
\end{aligned}
$$


The power spectrum of scalar perturbations in terms of the scalar field can also be written as

$$
\begin{aligned}
\mathcal{P}_{\mathcal{R}}= & \delta_{1} \phi^{\frac{1-m}{4-m}}\left(M^{-1}[S \phi]\right)^{\frac{(f-2)(m-3)(1+A)-(1-f)[(11-3 m)(1+A)+2 A(3-m)]}{(1+A)(4-m)}} \\
& \times\left[1-\frac{B}{1+A} \times\left(\frac{\kappa\left(M^{-1}[S \phi]\right)^{2(1-f)}}{3 \alpha^{2} f^{2}}\right)^{(1+\lambda)}\right]^{\frac{(3-m)[A+\lambda(1+A)]}{(4-m)(1+\lambda)(1+A)}},
\end{aligned}
$$

where $\delta_{1}$ is new constant which is given by

$$
\begin{aligned}
\delta_{1}= & \frac{\sqrt{3}}{4} \kappa(1+A)\left[\frac{C_{\phi}}{2 k C_{\gamma}(1+A)}\right]^{\frac{1}{4-m}} \\
& \times(1-f)^{\frac{m-3}{4-m}}(\alpha f)^{\frac{[(11-3 m)(1+A)+2 A(3-m)]-(3-m)(1+A)}{(1+A)(4-m)}} .
\end{aligned}
$$

The power spectrum may also be written as a function of the number of $e$-folds as follows:

$$
\begin{aligned}
& \mathcal{P}_{\mathcal{R}}(N) \\
& =\delta_{2}(M(J[N]))^{\frac{1-m}{4-m}}(J[N])^{\frac{(f-2)(m-3)(1+A)-(1-f)[(11-3 m)(1+A)+2 A(3-m)]}{(1+A)(4-m)}} \\
& \quad \times\left[1-\frac{B}{1+A}\left(\frac{\kappa(J[N])^{2(1-f)}}{3 \alpha^{2} f^{2}}\right)^{(1+\lambda)}\right]^{\frac{(3-m)[A+\lambda(1+A)]}{(4-m)(1+A)(1+\lambda)}},
\end{aligned}
$$

where the constant $\delta_{2}$ is defined as $\delta_{2}=\delta_{1} S^{\frac{m-1}{4-m}}$ and $J[N]$ is defined as $J[N]=\left[\frac{1+f(N-1)}{A f}\right]^{\frac{1}{f}}$. Additionally, the scalar spectral index $n_{s}$, defined by $n_{s}-1=\frac{d \ln \mathcal{P}_{\mathcal{R}}}{d \ln k}$, by using Eqs. (20) and (29), takes the form

$$
\begin{aligned}
& n_{S}=1 \\
& +\frac{(f-2)(m-3)(1+A)-(1-f)[(11-3 m)(1+A)+2 A(3-m)]}{\alpha f(1+A)(4-m)\left(M^{-1}[S \phi]\right)^{f}} \\
& +n_{2}+n_{3},
\end{aligned}
$$

where $n_{2}$ and $n_{3}$ are given by

$$
\begin{aligned}
n_{2}= & \left(\frac{1-m}{4-m}\right) \sqrt{\frac{2(1-f)}{\kappa \alpha f(1+A)}}\left(\frac{3 \alpha^{2} f^{2}}{\kappa}\right)^{-\frac{A}{2(1+A)}} \\
& \times\left(\frac{1}{\phi}\right)\left(M^{-1}[S \phi]\right)^{\frac{2 A(1-f)-f(1+A)}{2(1+A)}} \\
& \times\left[1-\frac{B}{1+A}\left(\frac{\kappa\left(M^{-1}[S \phi]\right)^{2(1-f)}}{3 \alpha^{2} f^{2}}\right)^{(1+\lambda)}\right]^{-\frac{A+\lambda(1+A)}{2(1+\lambda)(1+A)}}
\end{aligned}
$$

and

$$
\begin{aligned}
n_{3}= & -2 \frac{B}{1+A}\left(\frac{3-m}{4-m}\right)\left(\frac{A+\lambda(1+A)}{(1+A)}\right) \frac{(1-f)(\kappa / 3)^{1+\lambda}}{(\alpha f)^{3+2 \lambda}} \\
& \times\left(M^{-1}[S \phi]\right)^{2-3 f+2 \lambda(1-f)} \\
& \times\left[1-\frac{B}{1+A}\left(\frac{\kappa\left(M^{-1}[S \phi]\right)^{2(1-f)}}{3 \alpha^{2} f^{2}}\right)^{(1+\lambda)}\right]^{-1} .
\end{aligned}
$$

By using Eqs. (23) and (26), the scalar spectral index may also be written in terms of the number of $e$-folds $N$

$$
\begin{aligned}
& n_{S}=1 \\
& +\frac{(f-2)(m-3)(1+A)-(1-f)[(11-3 m)(1+A)+2 A(3-m)]}{(4-m)(1+A)[1+f(N-1)]} \\
& +n_{2}+n_{3},
\end{aligned}
$$

where $n_{2}$ and $n_{3}$ are defined as

$$
\begin{aligned}
n_{2}= & S\left(\frac{1-m}{4-m}\right) \sqrt{\frac{2(1-f)}{\kappa \alpha f(1+A)}}\left(\frac{3 \alpha^{2} f^{2}}{\kappa}\right)^{-\frac{A}{2(1+A)}} \\
& \times \frac{(J[N])^{\frac{2 A(1-f)-f(1+A)}{2(1+A)}}}{M(J[N])} \\
& \times\left[1-\frac{B}{1+A}\left(\frac{\kappa(J[N])^{2(1-f)}}{3 \alpha^{2} f^{2}}\right)^{(1+\lambda)}\right]^{-\frac{A+\lambda(1+A)}{2(1+\lambda)(1+A)}}
\end{aligned}
$$

and

$$
\begin{aligned}
n_{3}= & 2 \frac{B}{1+A}\left(\frac{3-m}{4-m}\right)\left(\frac{A+\lambda(1+A)}{(1+A)}\right) \\
& \times \frac{(1-f)(\kappa / 3)^{1+\lambda}}{\left.(\alpha f)^{3+2 \lambda}(J[N])\right)^{2-3 f+2 \lambda(1-f)}} \\
& \times\left[1-\frac{B}{1+A}\left(\frac{\kappa(J[N])^{2(1-f)}}{3 \alpha^{2} f^{2}}\right)^{(1+\lambda)}\right]^{-1} .
\end{aligned}
$$

Regarding tensor perturbations, these do not couple to the thermal background, so gravitational waves are only generated by quantum fluctuations, as in standard inflation [31]

$\mathcal{P}_{g}=8 \kappa\left(\frac{H}{2 \pi}\right)^{2}$

Having the tensor power spectrum, we may compute the tensor-to-scalar ratio $r=\mathcal{P}_{g} / \mathcal{P}_{\mathcal{R}}$, yielding the following:

$$
\begin{aligned}
r(\phi) & \\
= & \left(M^{-1}[S \phi]\right)^{\frac{(1-f)[(11-3 m)(1+A)+2 A(3-m)]-(f-2)(m-3)(1+A)-2(1-f)(1+A)(4-m)}{(1+A)(4-m)}} \\
& \times \frac{2 \kappa \alpha^{2} f^{2}}{\pi^{2} \delta_{1}} \phi^{\frac{m-1}{4-m}} \\
& \times\left[1-\frac{B}{1+A}\left(\frac{\kappa\left(M^{-1}[S \phi]\right)^{2(1-f)}}{3 \alpha^{2} f^{2}}\right)^{(1+\lambda)}\right]^{\frac{(m-3)[A+\lambda(1+A)]}{(4-m)(1+A)(1+\lambda)}} .
\end{aligned}
$$

Similarly, in terms of the number of $e$-folds $N$, the tensorto-scalar ratio becomes 


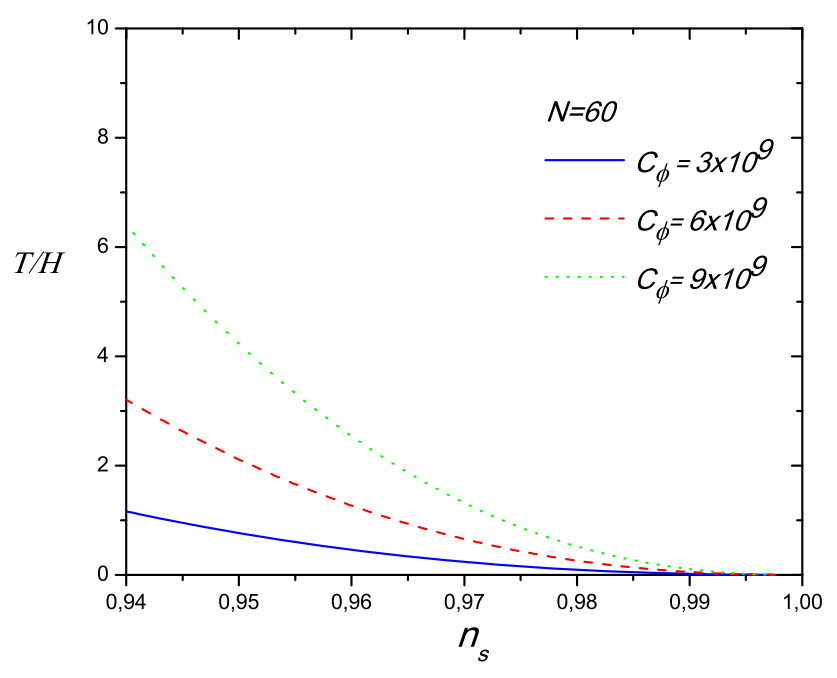

Fig. 1 Plots of $T / H$ as a function of the scalar spectral index $n_{s}$ (upper left) and $\Gamma / 3 H$ as a function of the scalar spectral index $n_{s}$ (upper right) for $N=60$. For both plots we have considered three different values of the parameter $C_{\phi}$ for the special case $m=3$, i.e., $\Gamma \propto T^{3} / \phi^{2}$, assuming the model evolves according to the weak dissipative regime. In both panels, the dotted, dashed, and solid lines correspond to the

$$
\begin{aligned}
r(N)= & (J[N]))^{\frac{(1-f)[(11-3 m)(1+A)+2 A(3-m)]-(f-2)(m-3)(1+A)-2(1-f)(1+A)(4-m)}{(1+A)(4-m)}} \\
& \times \frac{2 \kappa \alpha^{2} f^{2}}{\pi^{2} \delta_{2}}(M(J[N]))^{\frac{m-1}{4-m}} \\
& \times\left[1-\frac{B}{1+A}\left(\frac{\kappa(J[N]))^{2(1-f)}}{3 \alpha^{2} f^{2}}\right)^{(1+\lambda)}\right]^{\frac{(m-3)[A+\lambda(1+A)]}{(4-m)(1+A)(1+\lambda)}}
\end{aligned}
$$

In order to constrain our model, we must consider the essential condition for warm inflation, $T>H$, the condition for which the model evolves according to the weak regime, $R \ll 1$, and finally the Planck 2015 results [18], through the two-dimensional marginalized joint confidence contours for $n_{s}$ and $r$, at the 68 and 95\% CL. The upper left and upper right plots in Fig. 1 show the ratios $T / H$ and $\Gamma / 3 H$ as a functions of the scalar spectral index $n_{s}$ for the case $m=3$, i.e., $\Gamma(\phi, T)=C_{\phi} T^{3} / \phi^{2}$, respectively. To obtain both plots we used three different values for $C_{\phi}$ parameter and considered the following values characterizing the MCG: $A=0.0046, B=0.8289$ (by fixing $\rho_{m c g 0}=1$ ), and $\lambda=$ 0.1905 [84], and $C_{\gamma}=70$. In order to obtain numerical values for $T / H$ and $\Gamma / H$, for each value of $C_{\phi}$ we solve numerically Eqs. (29) and (31) for $\alpha$ and $f$, considering the observational values $\mathcal{P}_{\mathcal{R}} \simeq 2 \times 10^{-9}$ and $n_{s} \simeq 0.96$ [18], and fixing $N=60$. In this way, for $C_{\phi}=3 \times 10^{9}$, we obtain the values $\alpha=0.0062$ and $f=0.4703$, whereas, for $C_{\phi}=6 \times 10^{9}$, the solution is given by $\alpha=0.0043$ and $f=0.4702$. Finally, for $C_{\phi}=9 \times 10^{9}$, we find that $\alpha=0.0036$ and $f=0.4701$. From the upper left panel, we note that, for $C_{\phi}>3 \times 10^{9}$, the condition for warm inflation,

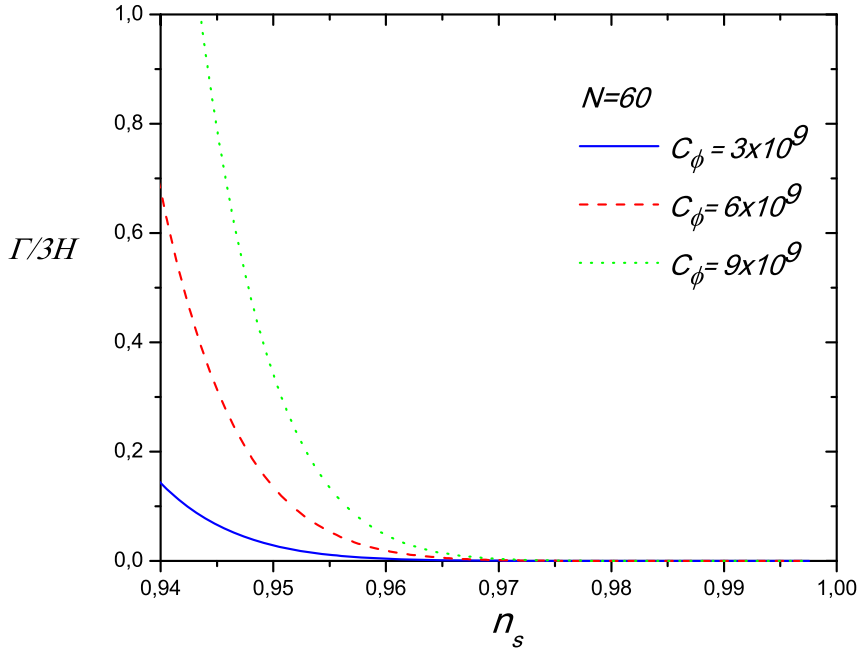

pairs $(\alpha=0.0062, f=0.4703),(\alpha=0.0043, f=0.4702)$, and $(\alpha=0.0036, f=0.4701)$, respectively. Lower left and lower right panels show $\Gamma / 3 H$ as a function of the scalar spectral index $n_{s}$ for $N=55$ and $N=70$, respectively. In these plots we have used the values $C_{\gamma}=70, A=0.0046, B=0.8289, \lambda=0.1905$, and $\kappa=1$

$T>H$, is always satisfied for the whole range considered for $n_{s}$. On the other hand, from the upper right panel, we note that, for $C_{\phi}<9 \times 10^{9}$, the model evolves according to the weak regime, $R \ll 1$. In this way, the condition for warm inflation gives us a lower limit on $C_{\phi}$ and, on the other hand, the condition for which the model evolves in agreement with the weak regime gives us an upper limit for $C_{\phi}$. Then, for the case $m=3$, the allowed range for $C_{\phi}$ become $3 \times 10^{9}<$ $C_{\phi}<9 \times 10^{9}$.

In addition, to see whether the change on the number of $e$ folds $N$ modifies the allowed range for $C_{\phi}$, firstly we consider $N=55$. We solve numerically Eqs. (29) and (31) for $\alpha$ and $f$, and considering the observational values $\mathcal{P}_{\mathcal{R}} \simeq 2 \times 10^{-9}$ and $n_{s} \simeq 0.96$ [18]. In order to make a direct comparison with the case $N=60$, we consider the same values already used for $C_{\phi}$. In this way, for $C_{\phi}=3 \times 10^{9}$, we obtain the values $\alpha=0.0015$ and $f=0.5237$, whereas, for $C_{\phi}=6 \times 10^{9}$, the solution is given by $\alpha=0.0011$ and $f=0.5237$. Finally, for $C_{\phi}=9 \times 10^{9}$, we find that $\alpha=0.0008$ and $f=0.5237$. Similarly, by fixing $N=70$ and for $C_{\phi}=3 \times 10^{9}$, we obtain the values $\alpha=0.5168$ and $f=0.2531$, whereas, for $C_{\phi}=6 \times 10^{9}$, the solution is given by $\alpha=0.4704$ and $f=0.2531$. Finally, for $C_{\phi}=9 \times 10^{9}$, we find that $\alpha=0.4456$ and $f=0.2531$. For $N=55$ and $N=70$, the essential condition for warm inflation to occur, through the plot $T / H$ as a function of $n_{s}$ (not shown) still imposes a lower limit for $C_{\phi}$ which is not modified with respect to $N=60$. However, from the left and right panels of Fig. 2, we infer that the condition for the model evolves according to weak regime, modifies the upper limit on $C_{\phi}$ for $N=55$ 


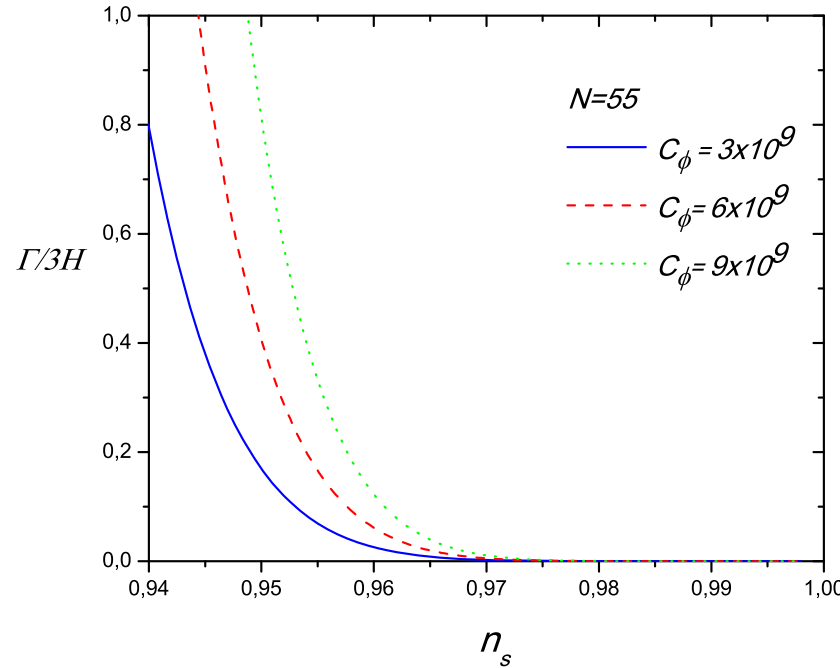

Fig. 2 Plots $\Gamma / 3 H$ as a function of the scalar spectral index $n_{s}$ for $N=55$ and $N=70$, respectively. For the left plot, the dotted, dashed, and solid lines correspond to the pairs $(\alpha=0.0015, f=0.5237)$, $(\alpha=0.0011, f=0.5237)$, and $(\alpha=0.0008, f=0.5237)$, respectively. Finally, for the right plot, the dotted, dashed, and solid lines

and $N=70$. In particular, for $N=55$, the new upper limit on $C_{\phi}$ becomes $6 \times 10^{9}$, which is lower than the previous found by fixing $N=60$. However, for $N=70$, the new upper bound becomes $10^{12}$, being greater than the already found for $N=60$. Then, for $N=55$ and $N=70$, the allowed ranges for $C_{\phi}$ are $3 \times 10^{9}<C_{\phi}<6 \times 10^{9}$ and $3 \times 10^{9}<C_{\phi}<\times 10^{12}$, respectively. Having in mind that the changes on $N$ imply a modification on the allowed ranges for $C_{\phi}$, particularly for the upper bound, although not significant, from as now we restrict ourselves to $N=60$.

It is interesting to mention that Planck data, through twodimensional marginalized joint confidence contours for $n_{s}$ and $r$, does not impose any constraint on our model for the special case $m=3$. In fact, for the several values considered before, the tensor-to-scalar ratio $r \sim 10^{-6}$ (figure not shown), being compatible with the Planck 2015 data, by considering the two-dimensional marginalized constraints at 68 and 95\% C.L. on the parameters $r$ and $n_{s}$ [18].

Finally, Fig. 3 shows the effective potential, given by Eq. (17), as a function of the inflaton field $\phi$ in the weak dissipative regime, for the case $m=3$ with $N=60$. Particularly, we have considered three different values of the parameter $C_{\phi}$, where the dotted, dashed, and solid lines correspond to the pairs $(\alpha=0.0062, f=0.4703),(\alpha=0.0043$, $f=0.4702)$, and $(\alpha=0.0036, f=0.4701)$, respectively. Inflation takes place as the field rolls down the potential, which tends asymptotically to zero as $\phi \rightarrow \infty$.

The left and right plots in Fig. 4 show the ratios $T / H$ and $\Gamma / 3 H$ as a functions of the scalar spectral index $n_{s}$ for the case $m=1$, i.e., $\Gamma(\phi, T)=C_{\phi} T$, respectively. To obtain

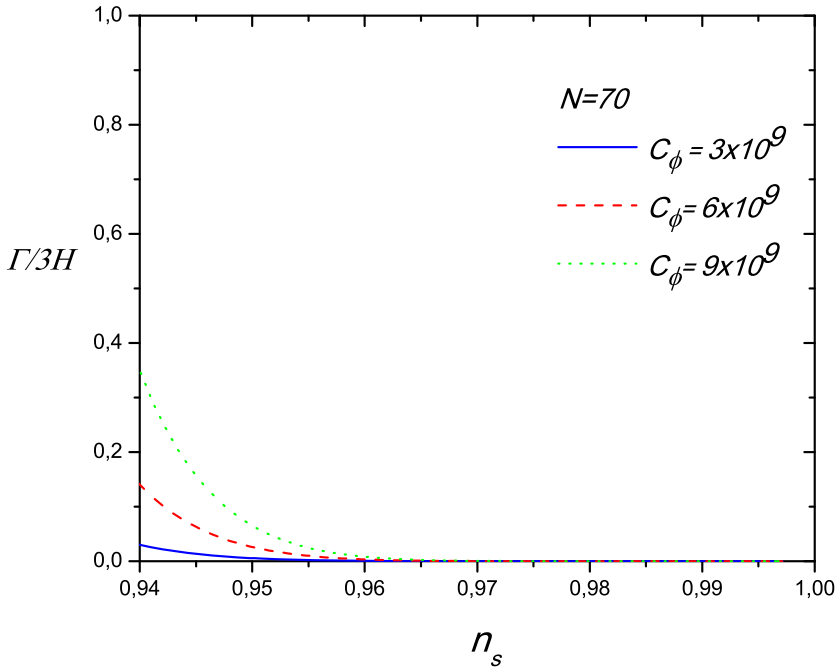

correspond to the pairs $(\alpha=0.5168, f=0.2531),(\alpha=0.4704$, $f=0.2531)$, and $(\alpha=0.4456, f=0.2531)$, respectively. For all plots we have used the values $C_{\gamma}=70, A=0.0046, B=0.8289$, $\lambda=0.1905$, and $\kappa=1$

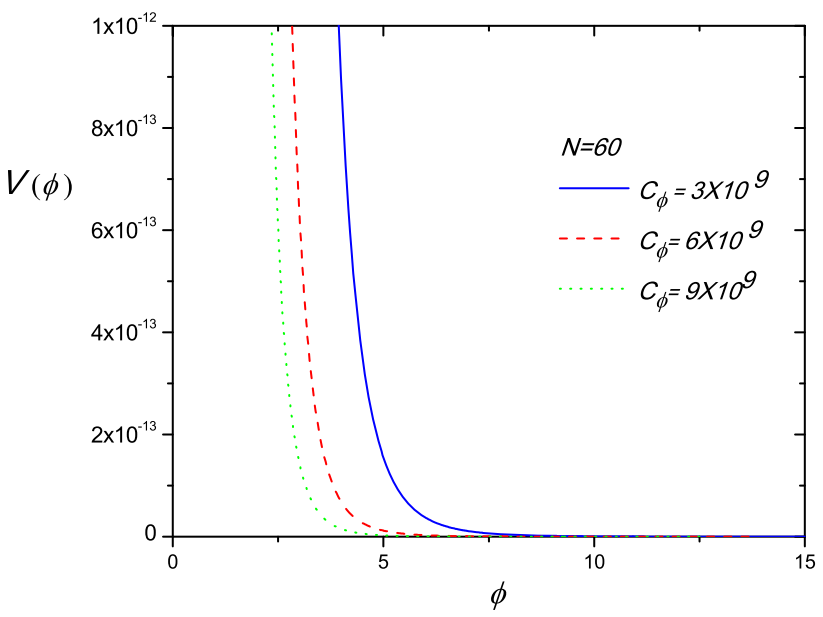

Fig. 3 Plot of the effective potential $V$ as a function of inflaton field $\phi$ for the case $m=3$ with $N=60$. For this plot we have considered three different values of the parameter $C_{\phi}$ for the special case $m=3$, i.e., $\Gamma \propto T^{3} / \phi^{2}$, assuming the model evolves according to the weak dissipative regime. The dotted, dashed, and solid lines correspond to the pairs $(\alpha=0.0062, f=0.4703),(\alpha=0.0043, f=0.4702)$, and ( $\alpha=0.0036, f=0.4701$ ), respectively. In addition, we have used the values $C_{\gamma}=70, A=0.0046, B=0.8289, \lambda=0.1905$, and $\kappa=1$

both plots we used three different values for $C_{\phi}$ parameter and considered the same values characterizing the MCG used for the case $m=3$, and $C_{\gamma}=70$. Following the same procedure as for the case $m=3$, for $C_{\phi}=3 \times 10^{-3}$ we obtain the values $\alpha=0.5168$ and $f=0.2531$, whereas, for $C_{\phi}=10^{-2}$, the solution is given by $\alpha=0.4704$ and $f=0.2532$. Finally, for $C_{\phi}=2 \times 10^{-2}$, we find that $\alpha=0.4456$ and $f=0.2533$. 
From the left panel, we note that for $C_{\phi}>3 \times 10^{-3}$, the condition for warm inflation, $\frac{T}{H}>1$, is always satisfied for the whole range considered for $n_{s}$. On the other hand, from the right panel, we note that, for $C_{\phi}<10^{-2}$, the models evolve according to the weak regime, $R \ll 1$. In this way, the condition for warm inflation gives us a lower limit on $C_{\phi}$ and, on the other hand, the condition for which the model evolves in agreement with the weak regime gives us an upper limit for $C_{\phi}$. Then, for the case $m=1$, the allowed range for $C_{\phi}$ is found to be $3 \times 10^{-3}<C_{\phi}<10^{-2}$. Again, the two-dimensional marginalized joint confidence contours for $n_{s}$ and $r$ dont impose any constraint on $C_{\phi}$. Additionally, for all the previous values, the tensor-to-scalar ratio $r \sim 10^{-7}$ (figure not shown), supported by Planck 2015 data.

As in previous cases, for $m=0$ and $m=-1$, the lower limit on $C_{\phi}$ corresponds to the minimum allowed value for which the essential condition for warm inflation, $\frac{T}{H}>1$, is satisfied, and on the other hand, the upper limit on $C_{\phi}$ correspond to the maximum allowed value for which the model evolves according to the weak regime $R \ll 1$. Specifically, for $m=0$, the lower limit on $C_{\phi}$ is given by $C_{\phi}=1.5 \times 10^{-9}$, for which we find numerically that $\alpha=0.8571$ and $f=0.2264$. Additionally, the upper limit on $C_{\phi}$ is found to be $C_{\phi}=1.5 \times 10^{-8}$. For this value we find numerically $\alpha=0.7514$ and $f=0.2266$. Finally, for $m=-1$, the lower limit on $C_{\phi}$ corresponds to $C_{\phi}=5.5 \times 10^{-16}$, for which we find numerically that $\alpha=1.1737$ and $f=0.2102$. The upper limit on $C_{\phi}$ is found to be $C_{\phi}=6.5 \times 10^{-15}$. For this value we find numerically $\alpha=1.0458$ and $f=0.2104$. Moreover, as in previous cases, we observe that the consistency relation $r\left(n_{s}\right)$ does not impose a constraint on $C_{\phi}$. In this way, for the weak dissipative regime, the constraints on our model are found only by considering the essential condition for warm inflation, $T>H$, and the condition for which the model evolves in agreement with the weak dissipative regime, $R \ll 1$.

\section{The strong dissipative regime}

In this section, we analyze the inflationary dynamics of our MCG model in the strong dissipative regime $\Gamma \gg 3 \mathrm{H}$. We can find the solution for the scalar field as a function of cosmic time by using Eqs. (13) and (18). Here, we study the solution for the two cases separately, for $m=3$ and $m \neq 3$.

\subsection{Special case $m=3$}

For the special case $m=3$, the scalar field as a function of cosmic time becomes

$\phi(t)-\phi_{0}=\exp \left(\frac{\tilde{M}[t]}{\tilde{S}}\right)$, where $\phi(t=0)=\phi_{0}$ is an integration constant. The quantity $\tilde{S}$ and the function $\tilde{M}[t]$ are given by

$$
\begin{aligned}
\tilde{S}= & 2^{-\frac{31}{8}} \frac{C_{\phi}{ }^{1 / 2}}{C_{\gamma}^{3 / 8}}(1+A)^{-7 / 8} \frac{(\kappa / 3)^{\frac{1}{8(1+A)}}}{(\alpha f)^{\frac{(3 A+5)}{8(1+A)}}} \\
& \times(1-f)^{-\frac{1}{8}}[(A(4+3 f)+5 f+2], \\
\tilde{M}[t]= & t^{\frac{A(4+3 f)+5 f+2}{8(1+\lambda)}}{ }_{2} F_{1} \\
& \times\left[\frac{A(4+3 f)+5 f+2}{16(1+A)(1-f)(1+\lambda)}, \frac{A+\lambda(1+A)}{8(1+A)(1+\lambda)},\right. \\
& 1+\frac{A(4+3 f)+5 f+2}{16(1+A)(1-f)(1+\lambda)}, \\
& \left.\times \frac{B}{1+A} \frac{\kappa t^{-2(f-1)(1+\lambda)}}{3 \alpha^{2} f^{2}}\right],
\end{aligned}
$$

respectively. One can find the Hubble rate for $m=3$ in terms of scalar field by utilizing Eqs. (19) and (35), thus,

$H(\phi)=\frac{\alpha f}{\left(\tilde{M}^{-1}[\tilde{S} \ln \phi]\right)^{1-f}}$.

For this case, the potential $V(\phi)$ leads to

$V(\phi) \approx\left[\left(\frac{3 \alpha^{2} f^{2}}{\kappa\left(\tilde{M}^{-1}[\tilde{S} \ln \phi]\right)^{2(1-f)}}\right)^{1+\lambda}-\frac{B}{1+A}\right]^{\frac{1}{(1+A)(1+\lambda)}}$.

The dissipative coefficient for $m=3$ in terms of the scalar field can be obtained by using Eqs. (18) and (35):

$$
\begin{aligned}
\Gamma(\phi)= & \delta_{3} \phi^{-2}\left(\tilde{M}^{-1}[\tilde{S} \ln \phi]\right)^{\frac{6 A(1-f)-3(2-f)(1+A)}{4(1+A)}}\left[1-\frac{B}{1+A}\right. \\
& \left.\times\left(\frac{\kappa\left(\tilde{M}^{-1}[\tilde{S} \ln \phi]\right)^{2(1-f)}}{3 \alpha^{2} f^{2}}\right)^{1+\lambda}\right]^{\frac{-3(A+\lambda(1+A))}{4(1+A)(1+\lambda)}}, \quad \text { (39) }
\end{aligned}
$$

here $\delta_{3}$ is a constant and attained the value $\delta_{3}=C_{\phi}$ $\left[\frac{\alpha f(1-f)}{2 \kappa C_{\gamma}(1+A)}\right]^{3 / 4}\left(\frac{3 \alpha^{2} f^{2}}{\kappa}\right)^{\frac{-3 A}{4(1+A)}}$. By combining Eqs. (19) and (35), we can find the relation for the number of $e$-folds $N$ as follows:

$N=\int_{t_{1}}^{t_{2}} H d t=\alpha\left(\left(\tilde{M}^{-1}\left[\tilde{S} \ln \phi_{2}\right]\right)^{f}-\left(\tilde{M}^{-1}\left[\tilde{S} \ln \phi_{1}\right]\right)^{f}\right)$.

Now, we shall study the cosmological perturbations for our model in the strong dissipative regime $R=\Gamma / 3 H>1$. For this regime, the scalar field fluctuation $\delta \phi$ is found to be $\delta \phi^{2} \simeq \frac{k_{F} T}{2 \pi^{2}}[32,33]$, where $k_{F}$ is a freeze-out wave number which is defined as $k_{F}=\sqrt{\Gamma H}=H \sqrt{3 R}>H$. In this way, the power spectrum of the scalar perturbation $\mathcal{P}_{\mathcal{R}}$ can be obtained by using Eqs. (16), (18), and (19): 


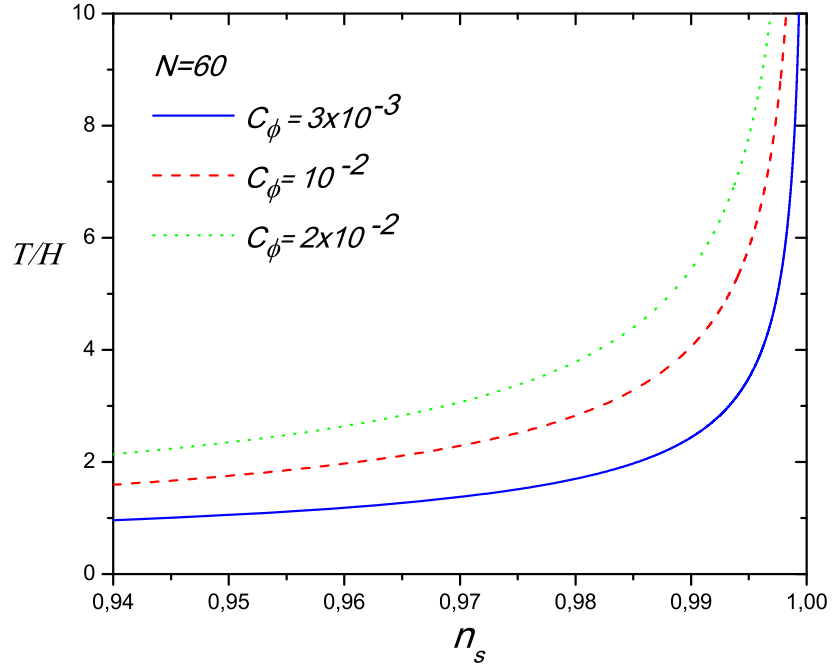

Fig. 4 Plots of $T / H$ as a function of the scalar spectral index $n_{S}$ (left) and $\Gamma / 3 H$ as a function of the scalar spectral index $n_{s}$ (right). For both plots we have considered three different values of the parameter $C_{\phi}$ for the special case $m=1$, i.e., $\Gamma \propto T$, assuming the model evolves according to the weak dissipative regime. In both panels, the dotted, dashed,

$$
\begin{aligned}
\mathcal{P}_{\mathcal{R}}= & \frac{H^{5 / 2} \Gamma^{1 / 2} T}{2 \pi^{2} \dot{\phi}^{2}} \\
= & \frac{\kappa(1+A)}{12 \pi^{2}} C_{\phi}{ }^{3 / 2}\left(\frac{3}{\kappa}\right)^{-\frac{3 A}{8(1+A)}}\left[\frac{3}{2 \kappa C_{\gamma}(1+A)}\right]^{\frac{11}{8}} \phi^{-3} \\
& \times H^{\frac{3(2+A)}{4(1+A)}}(-\dot{H})^{\frac{3}{8}}\left[1-\frac{B}{1+A}\left(\frac{3 H^{2}}{\kappa}\right)^{-(1+\lambda)}\right]^{-\frac{3(A+\lambda(1+A))}{8(1+A)(1+\lambda)}} .
\end{aligned}
$$

In addition, the power spectrum may also be expressed as a function of the scalar field $\phi$ for $m=3$ by using Eqs. (19), (35), and (41) as

$$
\begin{aligned}
\mathcal{P}_{\mathcal{R}} & =\delta_{4}\left(\tilde{M}^{-1}[\tilde{S} \ln \phi]\right) \frac{3(f-2)(1+A)-2(1-f)[6(1+A)-3 A]}{8(1+A)} \phi^{-3} \\
& \times\left[1-\frac{B}{1+A} \times\left(\frac{\kappa\left(\tilde{M}^{-1}[\tilde{S} \ln \phi]\right)^{2(1-f)}}{3 \alpha^{2} f^{2}}\right)^{1+\lambda}\right]^{-\frac{3(A+\lambda(1+A))}{8(1+A)(1+\lambda)}},
\end{aligned}
$$

where $\delta_{4}=\frac{\kappa(1+A)}{12 \pi^{2}} C_{\phi}{ }^{3 / 2}\left(\frac{3}{\kappa}\right)^{-\frac{3 A}{8(1+A)}}\left[\frac{3}{2 k C_{\gamma}(1+A)}\right]^{11 / 8}$ $(\alpha f)^{\frac{2[6(1+A)-3 A]+3(1+A)}{8(1+A)}}(1-f)^{3 / 8}$.

Similarly, in terms of the number of $e$-folds $N$, the power spectrum for $m=3$ becomes

$$
\begin{aligned}
\mathcal{P}_{\mathcal{R}}= & \delta_{4}(J[N])^{\frac{3(f-2)(1+A)-2(1-f)(6(1+A)-3 A)}{8(1+A)}} \exp \left(-\frac{3}{\tilde{S}} \tilde{M}(J[N])\right) \\
& \times\left[1-\frac{B}{1+A} \times\left(\frac{\kappa(J[N])^{2(1-f)}}{3 \alpha^{2} f^{2}}\right)^{1+\lambda}\right]^{\frac{-3(A+\lambda(1+A))}{8(1+A)(1+\lambda)}},
\end{aligned}
$$

where we use Eqs. (40) and (42). By using Eq. (42), we obtain the scalar spectral index $n_{s}$ as follows:

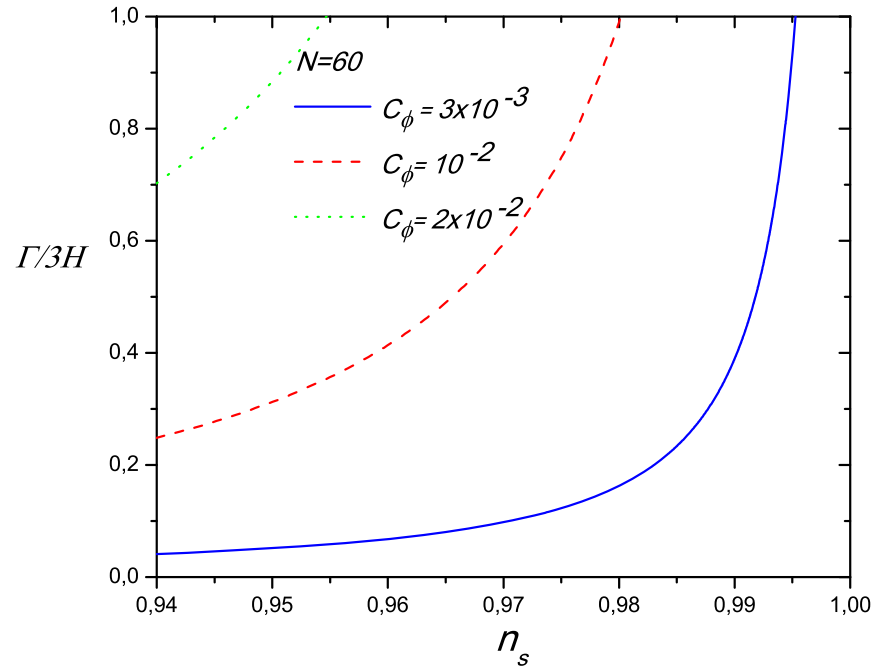

and solid lines correspond to the pairs $(\alpha=0.5168, f=0.2531)$, $(\alpha=0.4704, f=0.2532)$, and $(\alpha=0.4456, f=0.2533)$, respectively. In these plots we have used the values $C_{\gamma}=70, A=0.0046$, $B=0.8289, \lambda=0.1905$, and $\kappa=1$

$$
\begin{aligned}
n_{s}= & 1+\frac{3(f-2)(1+A)-2(1-f)(6(1+A)-3 A)}{8 \alpha f(1+A)} \\
& \times\left(\tilde{M}^{-1}[\tilde{S} \ln \phi]\right)^{-f}+n_{1}+n_{2},
\end{aligned}
$$

where

$$
\begin{aligned}
n_{1}= & -3\left(\frac{6}{\kappa(1+A)}\right)^{1 / 2}\left[\frac{3}{2 \kappa C_{\gamma}(1+A)}\right]^{-3 / 8}\left(\frac{3 \alpha^{2} f^{2}}{\kappa}\right)^{\frac{-A}{8(1+A)}}(1-f)^{1 / 8} \\
& \times \frac{(\alpha f)^{-3 / 8}}{C_{\phi}{ }^{1 / 2}\left(\tilde{M}^{-1}[\tilde{S} \ln \phi]\right)^{\frac{2 A(1-f)-(1+A)(4+3(f-2))}{8(1+A)}}\left[1-\frac{B}{1+A}\right.} \\
& \left.\left.\times\left(\frac{\kappa\left(\tilde{M}^{-1}[\tilde{S} \ln \phi]\right)^{2(1-f)}}{3 \alpha^{2} f^{2}}\right)\right]^{(1+\lambda)}\right]^{-\frac{A+\lambda(1+A)}{8(1+\lambda)(1+A)}} \\
n_{2}= & \frac{3(A+\lambda(1+A))}{4(1+A)} \frac{(\kappa / 3)^{1+\lambda}}{(\alpha f)^{3+2 \lambda}}(1-f)\left(\tilde{M}^{-1}[\tilde{S} \ln \phi]\right)^{2-3 f+2 \lambda(1-f)} \\
& \times\left[1-\frac{B}{1+A}\left(\frac{\kappa\left(\tilde{M}^{-1}[\tilde{S} \ln \phi]\right)^{2(1-f)}}{3 \alpha^{2} f^{2}}\right)^{1+\lambda}\right]^{-1}
\end{aligned}
$$

We can also express the scalar spectral index $n_{s}$ in terms of the number of $e$-folds $N$ as follows:

$$
\begin{aligned}
n_{s}= & 1+\frac{3(f-2)(1+A)-2(1-f)(6(1+A)-3 A)}{8 \alpha f(1+A)} \\
& \times(J[N])^{-f}+n_{1}+n_{2},
\end{aligned}
$$

where $n_{1}$ and $n_{2}$ are given by

$$
\begin{aligned}
n_{1}= & -3\left(\frac{6}{\kappa(1+A)}\right)^{1 / 2}\left[\frac{3}{2 \kappa C_{\gamma}(1+A)}\right]^{-3 / 8}\left(\frac{3 \alpha^{2} f^{2}}{\kappa}\right)^{\frac{-A}{8(1+A)}} \\
& \times(1-f)^{1 / 8} \frac{(\alpha f)^{-3 / 8}}{C_{\phi}^{1 / 2}}(J[N])^{\frac{2 A(1-f)-(1+A)(4+3(f-2))}{8(1+A)}}
\end{aligned}
$$




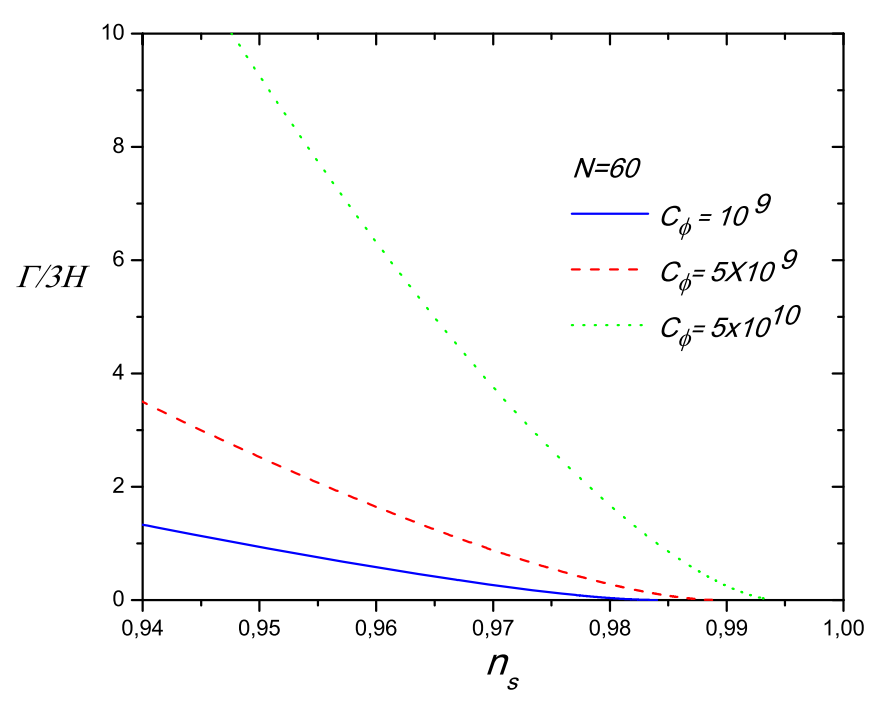

Fig. 5 Plots of $\Gamma / 3 H$ as a function of the scalar spectral index $n_{s}$ (left) and $T / H$ as a function of the scalar spectral index $n_{s}$ (right). For both plots we have considered three different values of the parameter $C_{\phi}$ for the special case $m=3$, i.e., $\Gamma \propto T^{3} / \phi^{2}$, assuming the model evolves according to the strong dissipative regime. In both panels, the

$$
\begin{aligned}
\times & {\left[1-\frac{B}{1+A}\left(\frac{\kappa(J[N])^{2(1-f)}}{3 \alpha^{2} f^{2}}\right)^{(1+\lambda)}\right]^{-\frac{A+\lambda(1+A)}{8(1+\lambda)(1+A)}} } \\
n_{2}= & \frac{3(A+\lambda(1+A))}{4(1+A)} \frac{(\kappa / 3)^{1+\lambda}}{(\alpha f)^{3+2 \lambda}(1-f)(J[N])^{2-3 f+2 \lambda(1-f)}} \\
& \times\left[1-\frac{B}{1+A} \times\left(\frac{\kappa(J[N])^{2(1-f)}}{3 \alpha^{2} f^{2}}\right)^{1+\lambda}\right]^{-1} .
\end{aligned}
$$

Regarding the tensor perturbations, the tensor-to-scalar ratio in terms of the scalar field for $m=3$ leads to

$$
\begin{aligned}
r= & \frac{2 \kappa}{\pi^{2} \delta_{4}}(\alpha f)^{2}\left(\tilde{M}^{-1}[\tilde{S} \ln \phi]\right)^{\frac{2(1-f)(6(1+A)-3 A)-3(f-2)(1+A)-16((1-f)(1+A)}{8(1+A)}} \phi^{3} \\
& \times\left[1-\frac{B}{1+A}\left(\frac{\kappa\left(\tilde{M}^{-1}[\tilde{S} \ln \phi]\right)^{2(1-f)}}{3 \alpha^{2} f^{2}}\right)^{1+\lambda}\right]^{\frac{3(A+\lambda(1+A))}{8(1+A)(1+\lambda)}} \cdot(46)
\end{aligned}
$$

In terms of the number of $e$-folds, the above expression turns out to be

$$
\begin{aligned}
r= & \frac{2 \kappa}{\pi^{2} \delta_{4}}(\alpha f)^{2}(J[N])^{\frac{2(1-f)(6(1+A)-3 A)-3(f-2)(1+A)-16((1-f)(1+A)}{8(1+A)}} \\
& \times \exp \left(3 \frac{\tilde{M}(J[N])}{\tilde{S}}\right) \\
& \times\left[1-\frac{B}{1+A}\left(\frac{\kappa(J[N])^{2(1-f)}}{3 \alpha^{2} f^{2}}\right)^{1+\lambda}\right]^{\frac{3(A+\lambda(1+A))}{8(1+A)(1+\lambda)}} .
\end{aligned}
$$

In order to constrain our model for this case, in a similar way to weak regime, we consider the essential condition for

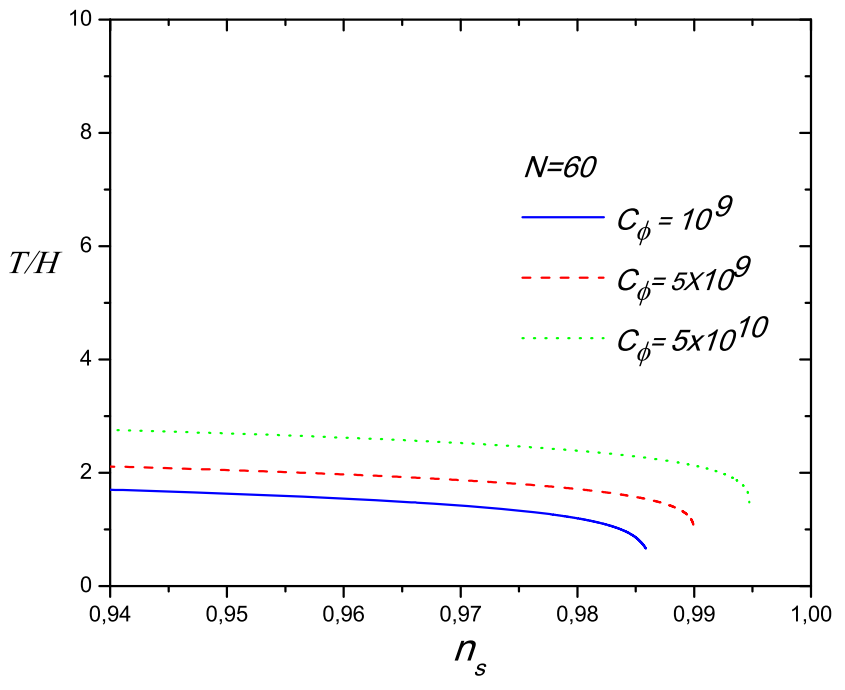

dotted, dashed, and solid lines correspond to the pairs ( $\alpha=0.0001$, $f=0.6451),(\alpha=0.0003, f=0.5756)$, and $(\alpha=0.0004$, $f=0.5313$ ), respectively. In these plots we have used the values $C_{\gamma}=70, A=0.0046, B=0.8289, \lambda=0.1905$, and $\kappa=1$

warm inflation, $T>H$, the condition for which the model evolves according to the weak regime, $R \ll 1$, and finally the two-dimensional marginalized joint confidence contours for $n_{s}$ and $r$, at the 68 and $95 \%$ CL, by Planck 2015 data [18]. The left and right plots in Fig. 5 show the ratios $\Gamma / 3 H$ and $T / H$ as functions of the scalar spectral index $n_{s}$ for the case $m=3$, i.e., $\Gamma(\phi, T)=C_{\phi} T^{3} / \phi^{2}$, respectively. To obtain both plots we used three different values for $C_{\phi}$ parameter and the values characterizing the MCG already used: $A=0.0046, B=0.8289$ (by fixing $\rho_{m c g 0}=1$ ), and $\lambda=0.1905$ [84], and $C_{\gamma}=70$. For each value of $C_{\phi}$ we solve Eqs. (29) and (31) numerically for $\alpha$ and $f$, considering the observational values $\mathcal{P}_{\mathcal{R}} \simeq 2 \times 10^{-9}$ and $n_{s} \simeq 0.96$ [18], by fixing $N=60$. In this way, for $C_{\phi}=10^{9}$, we obtain the values $\alpha=0.0001$ and $f=0.6451$, whereas, for $C_{\phi}=5 \times 10^{9}$, the solution is given by $\alpha=0.0003$ and $f=0.5756$. Finally, for $C_{\phi}=5 \times 10^{10}$, we find that $\alpha=0.0004$ and $f=0.5313$. From the left panel, we note that, for $C_{\phi}>10^{9}$, the model evolves according to the strong regime, $R \gg 1$. On the other hand, from the right panel, we note that, for $C_{\phi}>10^{9}$ the essential condition for warm inflation, $\frac{T}{H}>1$, is always satisfied. Then the condition for which the model evolves in agreement with the strong regime gives a lower limit on $C_{\phi}$. However, the essential condition for warm inflation does not impose any constraint. For the sake of comparison, we found numerically that the $\Gamma / 3 H$ and $T / H$ plots as a function of $n_{s}$ are not modified when we change the number of $e$-folds to $N=55$ and $N=70$. In this way, the lower limit already found does not change. On the other hand, Fig. 6 shows the trajectories in the $n_{s}-r$ plane 


$$
\begin{gathered}
\tilde{S}_{m}=2^{-\frac{(28+m)}{8}} \frac{C_{\phi}{ }^{1 / 2}}{C_{\gamma}{ }^{m} / 8}(1+A)^{-\frac{4+m}{8}} \frac{(\kappa / 3)^{\frac{4-m}{8(1+A)}}}{(\alpha f)^{\frac{4(1+A)+2 A(m-4)-(m-4)(1+A)}{8(1+A)}}(1-f)^{\frac{m-4}{8}}[A(4+f m)-f(8-m)+2 m-4],} \\
\tilde{M}_{m}[t]=t^{\frac{A(4+f m)-f(8-m)+2 m-4}{8(1+\lambda)}}{ }_{2} F_{1}\left[\frac{A(4+f m)-f(8-m)+2 m-4}{16(1+A)(1+\lambda)(1-f)}, \frac{(4-m)(A+\lambda(1+A))}{8(1+A)(1+\lambda)},\right. \\
\left.1+\frac{A(4+f m)-f(8-m)+2 m-4}{16(1+A)(1-f)(1+\lambda)}, \frac{B}{1+A} \frac{\kappa t^{-2(f-1)(1+\lambda)}}{3 \alpha^{2} f^{2}}\right],
\end{gathered}
$$

along with the two-dimensional marginalized constraints at 68 and 95\% C.L. on the parameters $r$ and $n_{s}$, by Planck 2015 data [18]. Here, we observe that, for $C_{\phi}>5 \times 10^{9}$, the model in the strong dissipative regime is supported by the observational data $\left(r \sim 10^{-8}\right)$. Then, for the special case $m=3$ with $N=60$, we were able to find only a lower limit for $C_{\phi}$.

\subsection{Special case $m \neq 3$}

The solution for the scalar field for the case $m \neq 3$ is found to be

$\varphi(t)-\varphi_{0}=\frac{\tilde{M}_{m}[t]}{\tilde{S}_{m}}$,

where $\varphi$ is a new scalar field, which is defined as $\varphi(t)=$ $\frac{2}{3-m} \phi(t)^{\frac{3-m}{2}}$. Also, $\tilde{S}_{m}$ and $\tilde{M}_{m}[t]$ are

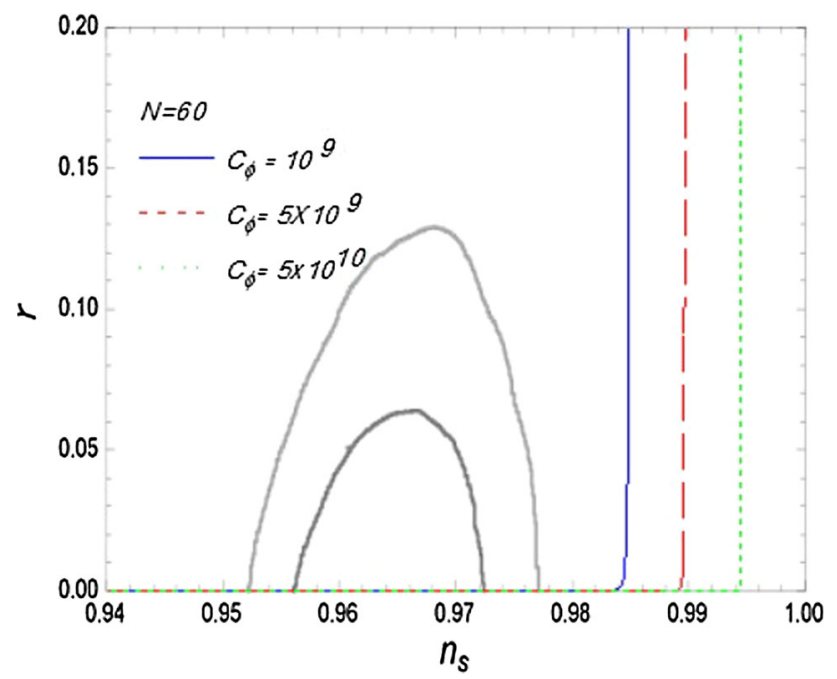

Fig. 6 Plot of the tensor-to-scalar ratio $r$ versus the scalar spectral index $n_{s}$ in the strong dissipative regime, for special case $m=3$, i.e., $\Gamma \propto T^{3} / \phi^{2}$. In addition, we have considered the two-dimensional marginalized joint confidence contours for $\left(n_{s}, r\right)$, at the 68 and $95 \%$ C.L., from the latest Planck data [18]. In this plot, the dotted, dashed, and solid lines correspond to the pairs ( $\alpha=0.0001, f=0.6451)$, $(\alpha=0.0003, f=0.5756)$, and $(\alpha=0.0004, f=0.5313)$, respectively. Moreover, we have used the values $C_{\gamma}=70, A=0.0046$, $B=0.8289, \lambda=0.1905$, and $\kappa=1$ respectively. Also, in this case, the Hubble parameter turns out to be

$H(\varphi)=\frac{\alpha f}{\left(\tilde{M}_{m}^{-1}\left[\tilde{S}_{m} \varphi\right]\right)^{1-f}}$.

For this case, the potential $V(\phi)$ takes the form

$V(\varphi) \approx\left[\left(\frac{3 \alpha^{2} f^{2}}{\kappa\left(\tilde{M}_{m}^{-1}\left[\tilde{S}_{m} \varphi\right]\right)^{2(1-f)}}\right)^{1+\lambda}-\frac{B}{1+A}\right]^{\frac{1}{(1+A)(1+\lambda)}}$

Moreover, the dissipative coefficient can be evaluated as

$$
\begin{aligned}
\Gamma(\phi)= & \delta_{m} \phi^{1-m}\left(\tilde{M}_{m}^{-1}\left[\tilde{S}_{m} \varphi\right]\right)^{\frac{2 m A(1-f)-m(2-f)(1+A)}{4(1+A)}}\left[1-\frac{B}{1+A}\right. \\
& \left.\times\left(\frac{\kappa\left(\tilde{M}_{m}^{-1}\left[\tilde{S}_{m} \varphi\right]\right)^{2(1-f)}}{3 \alpha^{2} f^{2}}\right)^{1+\lambda}\right]^{\frac{-m(A+\lambda(1+A))}{4(1+A)(1+\lambda)}}, \quad(52)
\end{aligned}
$$

where $\delta_{m}=C_{\phi}\left[\frac{\alpha f(1-f)}{2 \kappa C_{\gamma}(1+A)}\right]^{m / 4}\left(\frac{3 \alpha^{2} f^{2}}{\kappa}\right)^{\frac{-m A}{4(1+A)}}$.

The number of $e$-folds becomes

$N=\alpha\left(\left(\tilde{M}_{m}^{-1}\left(\tilde{S}_{m} \varphi_{2}\right]\right)^{f}-\left(\tilde{M}_{m}^{-1}\left[\tilde{S}_{m} \varphi_{1}\right]\right)^{f}\right)$.

For this case, the power spectrum turns out to be

$$
\begin{aligned}
\mathcal{P}_{\mathcal{R}}= & \tilde{\delta}_{m}\left(\tilde{M}_{m}^{-1}\left[\tilde{S}_{m} \varphi\right]\right) \frac{\frac{(3 m-6)(f-2)(1+A)-2(1-f)[3 A(2-m)+6(1+A))]}{8(1+A)}}{\phi^{\frac{3(1-m)}{2}}} \\
& \times\left[1-\frac{B}{1+A}\left(\frac{\kappa\left(\tilde{M}_{m}^{-1}\left[\tilde{S}_{m} \varphi\right]\right)^{2(1-f)}}{3 \alpha^{2} f^{2}}\right)^{1+\lambda}\right]^{\frac{(6-3 m)[A+\lambda(1+A)]}{8(1+A)(1+\lambda)}},
\end{aligned}
$$

where $\tilde{\delta}_{m}$ is defined as

$$
\begin{aligned}
\tilde{\delta}_{m}= & \frac{\kappa(1+A)}{12 \pi^{2}} C_{\phi}^{3 / 2}\left(\frac{3}{\kappa}\right)^{\frac{3 A(2-m)}{8(1+A)}}\left[\frac{3}{2 \kappa C_{\gamma}(1+A)}\right]^{\frac{3 m+2}{8}} \\
& \times(1-f)^{\frac{3 m-6}{8}} \times(\alpha f)^{\frac{2[3 A(2-m)+6(1+A)]+(3 m-6)(1+A)}{8(1+A)}} .
\end{aligned}
$$

In terms of the number of $e$-folds, we obtain

$$
\begin{aligned}
\mathcal{P}_{\mathcal{R}}= & \tilde{\gamma}_{m}(J[N])^{\frac{(3 m-6)(f-2)(1+A)-2(1-f)[3 A(2-m)+6(1+A))]}{8(1+A)}\left(\tilde{M}_{m}(J[N])\right)^{\frac{3(1-m)}{2}}} \\
& \times\left[1-\frac{B}{1+A}\left(\frac{\kappa(J[N])^{2(1-f)}}{3 \alpha^{2} f^{2}}\right)^{1+\lambda}\right]^{\frac{(6-3 m)(A+\lambda(1+A))}{8(1+A)(1+\lambda)}}, \quad \text { (55) }
\end{aligned}
$$


where $\tilde{\gamma}_{m}$ is defined as $\tilde{\gamma}_{m}=\left(\frac{1}{\tilde{\delta}_{m}}\right)^{\frac{3(1-m)}{2}}$. In this case, the scalar spectrum index $n_{s}$ becomes

$$
\begin{aligned}
& n_{s}=1 \\
& +\frac{(3 m-6)(f-2)(1+A)-2(1-f)[3 A(2-m)+6(1+A)]}{8 \alpha f(1+A)\left(\tilde{M}_{m}^{-1}\left[\tilde{S}_{m} \varphi\right]\right)^{f}} \\
& +n_{1_{m}}+n_{2_{m}},
\end{aligned}
$$

where

$$
\begin{aligned}
n_{1_{m}}= & \left(\frac{3(1-m)}{2}\right)\left(\frac{6}{\kappa(1+A)}\right)^{1 / 2} \\
& \times\left[\frac{3}{2 \kappa C_{\gamma}(1+A)}\right]^{-m / 8}(1-f)^{\frac{4-m}{8}} \phi^{\frac{m-3}{2}} \\
& \times\left(\frac{3 \alpha^{2} f^{2}}{\kappa}\right)^{\frac{(m-4) A}{8(1+A)}} \frac{(\alpha f)^{-m / 8}}{C_{\phi}^{1 / 2}} \\
& \times\left(\tilde{M}_{m}^{-1}\left[\tilde{S}_{m} \varphi\right]\right) \frac{-2 A(1-f)(m-4)-(1+A)(4+m(f-2))}{8(1+A)} \\
& \times\left[1-\frac{B}{1+A}\left(\frac{\kappa\left(\tilde{M}_{m}^{-1}\left[\tilde{S}_{m} \varphi\right]\right)^{2(1-f)}}{3 \alpha^{2} f^{2}}\right)^{(1+\lambda)}\right]^{\frac{(m-4)(A+\lambda(1+A))}{8(1+\lambda)(1+A)}} \\
n_{2_{m}}= & -\left(\frac{6-3 m}{4}\right)\left(\frac{A+\lambda(1+A)}{1+A}\right]^{1+\tilde{M}^{2}} \\
& \times \frac{(\kappa / 3)^{1+\lambda}(1-f)}{(\alpha f)^{3+2 \lambda}\left(\tilde{M}_{m}^{-1}\left[\tilde{S}_{m} \varphi\right]\right)^{2-3 f+2 \lambda(1-f)}} \\
& \times\left[1-\frac{B}{1+A}\left(\frac{\kappa\left(\tilde{M}_{m}^{-1}\left[\tilde{S}_{m} \varphi\right]\right)^{2(1-f)}}{3 \alpha^{2} f^{2}}\right)^{1+\lambda}\right]^{-1}
\end{aligned}
$$

The scalar spectral index in terms of $N$ becomes

$$
\begin{aligned}
& n_{s}=1 \\
& +\frac{(3 m-6)(f-2)(1+A)-2(1-f)[3 A(2-m)+6(1+A)]}{8 \alpha f(1+A)(J[N])^{f}} \\
& +n_{1_{m}}+n_{2_{m}},
\end{aligned}
$$

where

$$
\begin{aligned}
n_{1_{m}}= & \left(\frac{3(1-m)}{2}\right)\left(\frac{6}{\kappa(1+A)}\right)^{1 / 2} \\
& \times\left[\frac{3}{2 \kappa C_{\gamma}(1+A)}\right]^{-m / 8}(1-f)^{\frac{4-m}{8}} \phi^{\frac{m-3}{2}}\left(\frac{3 \alpha^{2} f^{2}}{\kappa}\right)^{\frac{(m-4) A}{8(1+A)}} \\
& \times \frac{(\alpha f)^{-m / 8}}{C_{\phi}{ }^{1 / 2}(J[N])} \\
& \times\left[1-\frac{B}{1+A}\left(\frac{\kappa(J[N])^{2(1-f)}}{3 \alpha^{2} f^{2}}\right)^{(1+\lambda)}\right]^{\frac{-2 A(1-f)(m-4)-(1+A)(4+m(f-2))}{8(1+\lambda)(1+A)}} \\
n_{2_{m}}= & -\frac{(6-3 m)[A+\lambda(1+A)]}{4(1+A)} \frac{(\kappa / 3)^{1+\lambda}}{(\alpha f)^{3+2 \lambda}}(1-f)(J[N])^{2-3 f+2 \lambda(1-f)} \\
& \times\left[1-\frac{B}{1+A}\left(\frac{\kappa(J[N])^{2(1-f)}}{3 \alpha^{2} f^{2}}\right)^{1+\lambda}\right]^{-1} .
\end{aligned}
$$

The tensor-to-scalar ratio takes the following form:

$$
\begin{aligned}
r= & \frac{2 \kappa}{\pi^{2} \tilde{\delta}_{m}}(\alpha f)^{2} \\
& \times\left(\tilde{M}_{m}^{-1}\left[\tilde{S}_{m} \varphi\right]\right)^{\frac{2(1-f)[3 A(2-m)+6(1+A)]-(3 m-6)(f-2)(1+A)-16((1-f)(1+A)}{8(1+A)}} \\
& \times \phi^{\frac{3(m-1)}{2}}\left[1-\frac{B}{1+A}\left(\frac{\kappa\left(\tilde{M}_{m}^{-1}\left[\tilde{S}_{m} \varphi\right]\right)^{2(1-f)}}{3 \alpha^{2} f^{2}}\right)^{1+\lambda}\right]^{\frac{(3 m-6)(A+\lambda(1+A))}{8(1+A)(1+\lambda)}}
\end{aligned}
$$

in terms of the number of $e$-folds,

$$
\begin{aligned}
r= & \frac{2 \kappa}{\pi^{2} \tilde{\delta}_{m}} \\
& \times(\alpha f)^{2}(J[N])^{\frac{2(1-f)[3 A(2-m)+6(1+A)]-(3 m-6)(f-2)(1+A)-16((1-f)(1+A)}{8(1+A)}} \\
& \times\left(\tilde{M}_{m}(J[N])\right)^{\frac{3(m-1)}{2}} \\
& \times\left[1-\frac{B}{1+A}\left(\frac{k(J[N])^{2(1-f)}}{3 \alpha^{2} f^{2}}\right)^{1+\lambda}\right]^{\frac{(3 m-6)(A+\lambda(1+A))}{8(1+A)(1+\lambda)}}
\end{aligned}
$$

For the case $m=1$, the condition for the model evolves according to strong dissipative regime, $R \gg 1$, gives us the lower limit on $C_{\phi}$, yielding $C_{\phi}=6 \times 10^{-2}$ (plot not shown). Additionally, for $C_{\phi}>6 \times 10^{-2}$ the condition for warm inflation, $\frac{T}{H}>1$, is always satisfied. Then we cannot find an upper limit on $C_{\phi}$ by considering the $T / H$ plot. Moreover, for $C_{\phi}>6 \times 10^{-2}$, the tensor-to-scalar ratio becomes $r \sim 0$, but the model is still supported by the last data of Planck, by considering the two-dimensional marginalized joint confidence contours for $\left(n_{s}, r\right)$, at the 68 and $95 \%$ C.L. (plot not shown). Then, for the case $m=1$, we were only able to find a lower limit for $C_{\phi}$, given by $C_{\phi}=6 \times 10^{-2}$.

For $m=-1$ and $m=0$, the predicted scalar spectral index is always greater than unity, being discarded by observations. This means that the inflaton decay ratios $\Gamma \propto \phi$ and $\Gamma \propto \frac{\phi^{2}}{T}$ are not suitable to describe a strong dissipative dynamics in the MCG scenario. It is interesting to mention that same behavior has been already reported in $[70,71,83]$.

\section{Conclusions}

In the present work we have studied warm inflationary dynamics inspired by the modified Chaplygin gas. We considered the inflationary expansion was driven by a standard scalar field with a generalized expression for its decay ratio $\Gamma=C_{\phi} T^{m} / \phi^{m-1}$, where $m=3,1,0,-1$, denotes several inflaton decay ratios studied in the literature. We have solved the background as well as perturbative dynamics considering the model to evolve according to the (1) strong and (2) strong dissipative regimes. For each dissipative regime, under the slow-roll approximation, we have found the expressions for the scalar power spectrum, scalar spectral index and tensorto-scalar ratio subsequently. Contrary to the standard cold inflation, in the warm inflation scenario it is not sufficient to consider only the constraints on the $r-n_{s}$ plane, but we 
also have to consider the essential condition for warm inflation $T>H$ and the conditions for the model evolves under the weak $(R \ll 1)$ or strong $(R \gg 1)$ dissipative regimes. In particular, for the weak dissipative regime, the condition for warm inflation and the condition for the model to evolve according to this regime, set the lower and upper limit for the dissipative parameter $C_{\phi}$, respectively. The Planck data, by considering the two-dimensional marginalized constraints at 68 and $95 \%$ C.L. on the parameters $r$ and $n_{s}$, does not impose any constraints on the model for this dissipative regime. However, the values for tensor-to-scalar ratio $r$ are compatible with current observational data. Regarding the strong dissipative, for the special case $m=3$, the condition for the model evolves under this regime and the Planck data, through the two-dimensional marginalized constraints on the parameters $r$ and $n_{s}$ set the lower and upper limits on the dissipative parameter $C_{\phi}$. However, for the case $m=1$, neither the condition for warm inflation nor the two-dimensional marginalized constraints on the parameters $r$ and $n_{s}$ impose constraints on $C_{\phi}$. The condition for the model evolves under the strong regime only sets a lower limit for this quantity. Finally, the two cases $m=0$ and $m=-1$ fail in describe a strong dissipative dynamics consistent with current data, since the predicted value for the scalar spectral index is always greater that unity. It is interesting to mention that the inflationary dynamics of our model under the strong regime predicts a value for the tensor-to-scalar ratio $r \sim 0$, but compatible with current data. We conclude that warm intermediate inflation inspired by the modified Chaplygin gas is compatible with current data for all the several inflaton decay ratios, parametrized by $m$, if we assume that our model evolves under the dissipative regime. However, if we assume that our model takes place in the strong dissipative regime, only the inflaton decay ratios yielding a dynamics compatible with current data correspond to $m=3$ and $m=1$.

Acknowledgements A. Jawad is thankful to the Higher Education Commission, Islamabad, Pakistan for its financial support under the grant No: 5412/Federal/NRPU/R\&D/HEC/2016 of NATIONAL RESEARCH PROGRAMME FOR UNIVERSITIES (NRPU). N.V. was supported by Comisión Nacional de Ciencias y Tecnología of Chile through FONDECYT Grant no. 3150490. Finally, the authors wish to thank the anonymous referee for her/his valuable comments, which have helped us to improve the presentation in our manuscript.

Open Access This article is distributed under the terms of the Creative Commons Attribution 4.0 International License (http://creativecomm ons.org/licenses/by/4.0/), which permits unrestricted use, distribution, and reproduction in any medium, provided you give appropriate credit to the original author(s) and the source, provide a link to the Creative Commons license, and indicate if changes were made. Funded by SCOAP ${ }^{3}$.

\section{References}

1. A. Guth, Phys. Rev. D 23, 347 (1981)

2. K. Sato, Mon. Not. R. Astron. Soc. 195, 467 (1981)
3. A.D. Linde, Phys. Lett. B 108, 389 (1982)

4. A.D. Linde, Phys. Lett. B 129, 177 (1983)

5. A. Albrecht, P.J. Steinhardt, Phys. Rev. Lett. 48, 1220 (1982)

6. V.F. Mukhanov, G.V. Chibisov, JETP Lett. 33, 532 (1981)

7. S.W. Hawking, Phys. Lett. B 115, 295 (1982)

8. A. Guth, S.-Y. Pi, Phys. Rev. Lett. 49, 1110 (1982)

9. A.A. Starobinsky, Phys. Lett. B 117, 175 (1982)

10. J.M. Bardeen, P.J. Steinhardt, M.S. Turner, Phys. Rev. D 28, 679 (1983)

11. D. Larson et al., Astrophys. J. Suppl. 192, 16 (2011)

12. C.L. Bennett et al., Astrophys. J. Suppl. 192, 17 (2011)

13. N. Jarosik et al., Astrophys. J. Suppl. 192, 14 (2011)

14. G. Hinshaw et al. [WMAP Collaboration], Astrophys. J. Suppl. 208, 19 (2013)

15. P.A.R. Ade et al. [Planck Collaboration], Astron. Astrophys. 571, A16 (2014)

16. P.A.R. Ade et al. [Planck Collaboration], Astron. Astrophys. 571, A22 (2014)

17. P.A.R. Ade et al. [Planck Collaboration], Astron. Astrophys. 594, A13 (2016)

18. P.A.R. Ade et al. [Planck Collaboration], Astron. Astrophys. 594, A20 (2016)

19. L. Kofman, A.D. Linde, A.A. Starobinsky, Phys. Rev. Lett. 73, 3195 (1994)

20. L. Kofman, A.D. Linde, A.A. Starobinsky, Phys. Rev. D 56, 3258 (1997)

21. M.A. Amin, M.P. Hertzberg, D.I. Kaiser, J. Karouby, Int. J. Mod. Phys. D 24, 1530003 (2014)

22. I.G. Moss, Phys. Lett. B 154, 120 (1985)

23. A. Berera, Phys. Rev. Lett. 75, 3218 (1995)

24. A. Berera, Phys. Rev. D 55, 3346 (1997)

25. Y. Zhang, JCAP 0903, 023 (2009)

26. M. Bastero-Gil, A. Berera, R.O. Ramos, J.G. Rosa, JCAP 1301, $016(2013)$

27. L.M.H. Hall, I.G. Moss, A. Berera, Phys. Rev. D 69, 083525 (2004)

28. A. Berera, Phys. Rev. D 54, 2519 (1996)

29. A. Berera, L.Z. Fang, Phys. Rev. Lett. 74, 1912 (1995)

30. A. Berera, Nucl. Phys. B 585, 666 (2000)

31. A.N. Taylor, A. Berera, Phys. Rev. D 62, 083517 (2000)

32. A. Berera, I.G. Moss, R.O. Ramos, Rep. Prog. Phys. 72, 026901 (2009)

33. M. Bastero-Gil, A. Berera, Int. J. Mod. Phys. A 24, 2207 (2009)

34. A. Riess et al., Astron. J. 116, 1009 (1998)

35. P. Garnavich et al., Astrophys. J. 509, 74 (1998)

36. S. Permutter et al., Astrophys. J. 575, 565 (1999)

37. S. Permutter et al., Astrophys. J. 598, 102 (2003)

38. P.J.E. Peebles, B. Ratra, Rev. Mod. Phys. 75, 559 (2003)

39. B. Ratra, P.J.E. Peebles, Phys. Rev. D 37, 3406 (1988)

40. R.R. Caldwell, R. Dave, P.J. Steinhardt, Phys. Rev. Lett. 80, 1582 (1998)

41. M. Sami, T. Padmanabhan, Phys. Rev. D 67, 083509 (2003)

42. C. Armendariz-Picon, V. Mukhanov, P.J. Steinhardt, Phys. Rev. D 63, $103510(2001)$

43. T. Chiba, Phys. Rev. D 66, 063514 (2002)

44. R.J. Scherrer, Phys. Rev. Lett. 93, 011301 (2004)

45. A. Sen, J. High Energy Phys. 04, 048 (2002)

46. A. Sen, J. High Energy Phys. 07, 065 (2002)

47. G.W. Gibbons, Phys. Lett. B 537, 1 (2002)

48. R.R. Caldwell, Phys. Lett. B 545, 23 (2002)

49. E. Elizade, S. Nojiri, S. Odintsov, Phys. Rev. D 70, 043539 (2004)

50. J.M. Cline, S. Jeon, G.D. Moore, Phys. Rev. D 70, 043543 (2004)

51. A. Kamenshchik, U. Moschella, V. Pasquier, Phys. Lett. B 511, $265(2001)$

52. M. Li, Phys. Lett. B 603, 1 (2004)

53. M.C. Bento, O. Bertolami, A.A. Sen, Phys. Rev. D 70, 083519 (2004) 
54. M.C. Bento, O. Bertolami, A.A. Sen, Phys. Lett. B 575, 172 (2003)

55. M. Makler, S. Quinet de Oliveira, I. Waga, Phys. Lett. B 555, 1 (2003)

56. L. Amendola, F. Finelli, C. Burigana, D. Carturan, JCAP 0307, 005 (2003)

57. H.B. Benaoum, arXiv:hep-th/0205140

58. J. Lu, L. Xu, J. Li, B. Chang, Y. Gui, H. Liu, Phys. Lett. B 662, 87 (2008)

59. U. Debnath, A. Banerjee, S. Chakraborty, Class. Quantum Gravity 21, 5609 (2004)

60. S. Silva e Costa, M. Ujevic, A. Ferreira dos Santos, Gen. Relativ. Grav. 40, 1683 (2008)

61. O. Bertolami, V. Duvvuri, Phys. Lett. B 640, 121 (2006)

62. R. Herrera, S. del Campo, C. Campuzano, J. Cosmol. Astropart. Phys. 10, 009 (2006)

63. S. del Campo, R. Herrera, D. Pavon, Phys. Rev. D 75, 083518 (2007)

64. S. del Campo, R. Herrera, Phys. Lett. B 653, 122 (2007)

65. M.A. Cid, S. del Campo, R. Herrera, J. Cosmol. Astropart. Phys. 10, 005 (2006)

66. J.C.B. Sanchez, M. Bastero-Gil, A. Berera, K. Dimopoulos, Phys. Rev. D 77, 123527 (2008)

67. R. Herrera, Phys. Rev. D 81, 123511 (2010)

68. R. Herrera, E. San Martin, Eur. Phys. J. C 71, 1701 (2011)

69. M.R. Setare, V. Kamali, Phys. Lett. B 726, 56 (2013)

70. R. Herrera, M. Olivares, N. Videla, Eur. Phys. J. C 73, 2295 (2013)

71. R. Herrera, M. Olivares, N. Videla, Phys. Rev. D 88, 063535 (2013)

72. A. Jawad, S. Rani, Commun. Theor. Phys. 65, 653 (2016)
73. A. Jawad, S. Rani, S. Mohsaneen, Astrophys. Space Sci. 361, 158 (2016)

74. A. Jawad, S. Rani, S. Mohsaneen, Eur. Phys. J. Plus 131, 234 (2016)

75. A. Jawad, S. Butt, S. Rani, Eur. Phys. J. C 76, 274 (2016)

76. A. Jawad, A. Ilyas, S. Rani, Astropart. Phys. 81, 61-71 (2016)

77. A. Jawad, A. Ilyas, S. Rani, Int. J. Mod. Phys. D 26, 1750031 (2017)

78. K. Bamba, S.D. Odintsov, Eur. Phys. J. C 76, 18 (2016)

79. K. Bamba, S.D. Odintsov, P.V. Tretyakov, Eur. Phys. J. C 75, 344 (2015)

80. K. Bamba, S.D. Odintsov, Symmetry 7, 220 (2015)

81. K. Bamba et al., Phys. Rev. D 90, 124061 (2014)

82. K. Bamba, S. Nojiri, S.D. Odintsov, Phys. Lett. B 731, 257 (2014)

83. R. Herrera, N. Videla, M. Olivares, Eur. Phys. J. C 76, 35 (2016)

84. B.C. Paul, P. Thakur, A. Beesham, Astrophys. Space Sci. 361(10), $336(2016)$

85. T. Barreiro, A.A. Sen, Phys. Rev. D 70, 124013 (2004)

86. F. Lucchin, S. Matarrese, Phys. Rev. D 32, 1316 (1985)

87. J.D. Barrow, Phys. Lett. B 235, 40 (1990)

88. J.D. Barrow, A.R. Liddle, Phys. Rev. D 47(12), R5219 (1993)

89. A.D. Rendall, Class. Quantum Gravity 22, 1655 (2005)

90. J.D. Barrow, A.R. Liddle, C. Pahud, Phys. Rev. D 74, 127305 (2006)

91. J.D. Barrow, M. Lagos, J. Magueijo, Phys. Rev. D 89(8), 083525 (2014)

92. D. Veberic, Comput. Phys. Commun. 183, 2622 (2012)

93. D.H. Lyth, A.R. Liddle, The primordial density perturbation: Cosmology, inflation and the origin of structure, (Cambridge, UK: Cambridge University Press. 2009), p. 497 